\title{
Review on improved seismic imaging with closure phase
}

\author{
Gerard Schuster ${ }^{1}$, Yunsong Huang ${ }^{1}$, Sherif M. Hanafy ${ }^{1}$, Min Zhou ${ }^{2}$, \\ Jianhua $\mathrm{Yu}^{2}$, Ola Alhagan ${ }^{3}$, and Wei Dai ${ }^{1}$
}

\begin{abstract}
The timing and amplitudes of arrivals recorded in seismic traces are influenced by velocity variations all along the associated raypaths. Consequently, velocity errors far from the target can lead to blurred imaging of the target body. To partly remedy this problem, we comprehensively reviewed inverting differential traveltimes that satisfied the closurephase condition. The result is that the source and receiver statics are completely eliminated in the data and velocities far from the target do not need to be known. We successfully used the phase closure equation for traveltime tomography, refraction statics, migration, refraction tomography, and earthquake location, all of which demonstrated the higher resolution achievable by processing data with differential traveltimes rather than absolute traveltimes. More generally, the stationary version of the closure-phase equation is equivalent to Fermat's principle and can be derived from the equations of seismic interferometry. In summary, the general closure-phase equation is the mathematical foundation for approximately redatuming sources and/or receivers to the target of interest without the need to accurately know the statics or the velocity model away from the target.
\end{abstract}

\section{INTRODUCTION}

Optical interferometry is a technique that uses the interference of photons to give high-resolution estimates of distances, displacements, temperature gradients, and other physical parameters (Hariharan, 2010). For example, a rainbow of colors is seen in an oil slick on water because sunlight reflections from the top of the oil-air interfere with those from the bottom to give an interference pattern that depends on wavelength (i.e., color). This idea is exploited by industrial engineers in using optical interference patterns to precisely measure the curvature of an optical lens.

Another use for interferometry is to image stellar objects. Here, the photons received by two microwave antennae with separation distance $D$ are combined to give an interference pattern that has a resolution theoretically equal to that of a single antenna with width $D$. This not only increases the resolution capabilities of small telescopes but, using the closure-phase equation, it can also eliminate image distortions due to atmospheric disturbances in radio and optical astronomy (Jennison, 1958; Cornwell, 1989; Wohlleben et al., 1991). For example, Figure 1 shows a star emitting photons that fall on a three-hole mask placed in front (to the left) of an aberrationfree lens, which in turn focuses the incident light waves onto the image plane. A unique atmospheric turbulence is assumed in front of each pinhole. Photons from pinhole $i$ interfere with those propagating from $j$ to give, after processing (Rhodes, 2009), the corrupted phase $\phi_{i j}=\psi_{i j}+\theta_{i}-\theta_{j}$ at the image plane. This recorded phase $\phi_{i j}$ is a weighted sum of the object's phase interference $\psi_{i j}$ and the turbulence-induced phase distortions $\theta_{i}$ and $\theta_{j}$ at the $i$ and $j$ pinholes, respectively (for an exploration seismologist, the phase distortion $\theta_{i}$ is analogous to the receiver statics at the $i$ th receiver).

The goal is to eliminate the phase distortions $\theta_{i}$ s to obtain the object phases $\psi_{i j}$. This can be achieved with three pinholes by the weighted closure-phase summation of $\phi_{i j}$ over unique pairs of indices, giving

$$
\phi_{123}=\overbrace{\phi_{12}+\phi_{23}-\phi_{13}}^{\text {corrupted phases }}=\overbrace{\psi_{12}+\psi_{23}-\psi_{13}}^{\text {object phases }},
$$

which yields the sum of object phases uncorrupted by turbulenceinduced distortions. This sum of uncorrupted object phases can be combined with recorded phases from other masks with different

Manuscript received by the Editor 23 August 2013; revised manuscript received 2 March 2014; published online 13 August 2014.

${ }^{1}$ King Abdullah University of Science and Technology, Division of Physical Science and Engineering, Center for Subsurface Imaging and Fluid Modeling, Thuwal, Saudi Arabia. E-mail: gerard.schuster@kaust.edu.sa; yunsong.huang@kaust.edu.sa; sh3m3@yahoo.com; wei.dai@utah.edu.

${ }^{2}$ BP America, Inc., Houston, Texas, USA. E-mail: min.zhou@bp.com; jianhua.yu@bp.com.

${ }^{3}$ King Khalid University, College of Science, Physics Department, Abha, Saudi Arabia. E-mail: olahagan@hotmail.com.

(C) 2014 Society of Exploration Geophysicists. All rights reserved. 
numbers and arrangements of pinholes to estimate the individual phases $\psi_{i j}$ (see Rhodes, 2009).

In seismology, the atmospheric disturbances are analogous to unexplained velocity perturbations in the earth velocity model. To explain rather than eliminate such anomalies, earthquake seismologists (Lay and Wallace, 1995; Wright, 2008) invert for a station correction that accounts for the inaccuracies of the velocity model structure along a travel path and beneath a station; the inversion procedure is known as the joint hypocentral determination (JHD) (Douglas, 1967). Without these station corrections, the hypocentral location can be grossly misestimated (Dziewonski and Anderson, 1983; Wright, 2008). If the velocity anomalies are localized beneath the recording stations, then exploration seismologists define them as static anomalies and the corresponding delays in the reflection arrivals are known as static delays (Sherriff [1991] defines static corrections, often shortened to statics, as "corrections applied to seismic data to compensate for the effects in elevation, weathering thickness, weathering velocity, or reference to a datum").

To mitigate the hypocenter errors caused by velocity anomalies, seismologists developed the double-difference (DD) method (Waldhauser and Ellsworth, 2000; Zhang and Thurber, 2003; Michelini and Lomax, 2004). Their procedure is akin to a partial closurephase correction in which the traveltimes (phases in equation 1) of either P- or S-waves from neighboring events recorded at the same station are subtracted from one another to give the differential traveltimes. These differential traveltimes are used as input into a JHD procedure to estimate hypocenters. The benefits are elimination of the localized receiver statics and less sensitivity to velocity errors for hypocenter estimation. A similar procedure, named interferometric traveltime tomography (ITT), was developed for exploration seismology by Zhou and Schuster (2000) to eliminate source and receiver statics in traveltime tomography. Unlike the DD method, ITT eliminates source and receiver statics that might corrupt the accuracy of velocity tomograms. For migration images, Sheley and Schuster (2003) use a partial closure-phase correction to reduce defocusing in migration images caused by errors in the migration velocity. The closure-phase correction is also used by $\mathrm{Yu}$ (2000) and Sheng et al. (2005) to eliminate statics in stacked reflections.

Until now, it has not been recognized that the above methods are related to one another beyond the fact that picked traveltimes are used to time shift traces. We now unify all of these data-driven phase-shift procedures under one general closure-phase equation. This general equation also leads to several new applications of clo-

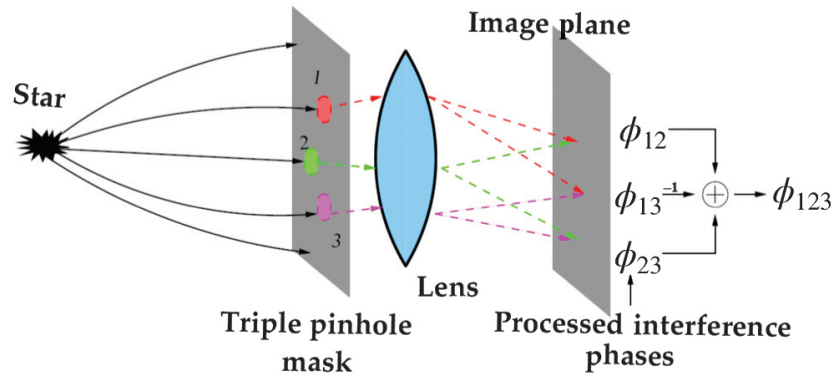

Figure 1. Imaging system with a three-hole mask in front (to the left) of an optical lens; the lens is in the far-field region of the star. Atmospheric turbulence is assumed just in front of the three pinholes, and its distorting effects on the image can be eliminated by the closure-phase formula 1 . sure phase in seismology, including the prediction of far-offset refraction traveltimes from shorter offset refraction traveltimes and the corresponding potential for enhancing the signal-to-noise ratio $(\mathrm{S} / \mathrm{N})$ of far-offset refractions, diffractions, and surface waves. We also show that the stationary version of the closure-phase equation is a restatement of Fermat's principle and can be derived from the reciprocity equations of correlation and convolution types. This means that seismic interferometry is the underlying mathematical foundation for the equations of the closure phase.

The rest of this review is organized as follows. First, we review the generalized equation of closure phase for seismology. Examples are presented for mitigating statics-induced inaccuracies in traveltime tomograms, hypocenter locations, and stacked reflection sections. Then, we define the stationary closure-phase equation and show how it can be used to increase the $\mathrm{S} / \mathrm{N}$ of head waves and diffractions. Numerical examples are presented for estimating longoffset traveltimes in refraction data and for the extraction of diffractions in noisy shot gathers. The appendices link the stationary closure-phase equation with the reciprocity equations of correlation and convolution types and Fermat's principle. Finally, a summary and discussion are presented.

\section{CLOSURE-PHASE APPLICATIONS IN SEISMOLOGY}

To derive the closure-phase equations of seismology, assume the source-receiver configuration in Figure $2 \mathrm{a}$, where the recorded propagation time from the $i$ th source to the $k$ th receiver is defined as

$$
T_{i k}=t_{i k}+\tau_{i}^{\mathrm{src}}+\tau_{k}^{\mathrm{rec}}
$$

where $\tau_{i}^{\mathrm{src}}$ and $\tau_{k}^{\mathrm{rec}}$ represent, respectively, the source and receiver statics, and $t_{i k}$ defines the no-statics wave-propagation time from the $i$ th source to the $k$ th receiver. The event of interest can be of any type, such as a refraction, diffraction, or reflection, and the goal is to write a weighted combination of traveltimes so that all of the statics terms are eliminated.

The static terms in $T_{i k}$ can be eliminated by combining the traveltimes, similar to combining the phases in equation 1 , to give the

\section{Three- and four-point closure phase formulas}

$$
\phi_{i j k l}=T_{i k}-T_{i l}+T_{j l}-T_{j k} \quad \phi_{i j k}=T_{i k}-T_{j k}
$$

a) Refraction tomography b) Reflection tomography,

c) Double differencing
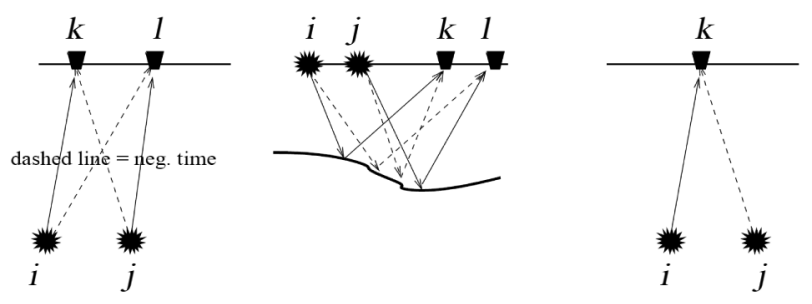

Figure 2. Four-point closure-phase formula eliminates the source and receiver statics for (a) transmission traveltime tomography and (b) reflection traveltime tomography, migration, and reflection stacks. (c) The three-point formula eliminates the receiver statics for the DD method. The star symbol denotes a seismic source, and the solid quadrilateral denotes a receiver; dashed (solid) rays denote raypaths where the traveltime is scaled by the value $-1(+1)$. 
four-point (there are two receiver and two source points to give a total of four distinct starting and end points for the Figure $2 a$ and $2 b$ rays) closure-phase equation:

$$
\begin{aligned}
\Phi_{i j k l} & =\overbrace{T_{i k}-T_{i l}+T_{j l}-T_{j k}}^{\text {corrupted times }}, \\
& =\overbrace{t_{i k}-t_{i l}+t_{j l}-t_{j k}}^{\text {object times }} .
\end{aligned}
$$

This is a total closure-phase formula because the source and receiver statics are eliminated, which means that only pairs of solid and dashed rays intersect any source or receiver location. In Figure 2 the solid and dashed rays correspond to positive and negative traveltimes, respectively.

In contrast to the total closure-phase equation 3, the three-point closure-phase formula

$$
\begin{aligned}
\Phi_{i j k} & =T_{i k}-T_{j k} \\
& =\overbrace{t_{i k}-t_{j k}}^{\text {object times }}+\overbrace{\tau_{i}^{\text {src }}-\tau_{j}^{\text {src }}}^{\text {source corruption }},
\end{aligned}
$$

only eliminates the receiver statics, and so it is labeled a partial closure-phase formula. The associated ray diagram for the above equation is shown in Figure 2c, where the differential traveltimes $\Phi_{i j k}$ are inverted to decrease errors in hypocenter location (Waldhauser and Elsworth, 2000) and velocity tomograms (Zhang and Thurber, 2003; Michelini and Lomax, 2004).

More generally, the generalized closure-phase formula is defined as

$$
\Phi_{B}=\sum_{p=1}^{P} \overbrace{\left(\bar{T}_{\alpha_{p}}-T_{\beta_{p}}\right)}^{\text {differential traveltimes }},
$$

where $\alpha_{p}$ and $\beta_{p}$ represent the $p$ th index pairs such as $j k$ or $l k$. This sum is over the specified differential traveltimes $\bar{T}_{\alpha_{i}}-T_{\beta_{j}}$, and $B$ is the set of independent indices. As an example, set $P=1, \alpha_{1}=i k, \beta_{1}=j k, \bar{T}_{\alpha_{1}}=T_{i k}$, and $B=[i j k]$ to obtain the three-point formula in equation 4. Similarly, setting $P=2$, $\alpha_{1}=i k, \alpha_{2}=j l, \beta_{1}=i l, \beta_{2}=j k, B=i j k l$, and $\bar{T}_{\alpha_{i}}=T_{\alpha_{i}}$ yields the four-point formula 3 . The defining constraint is that the sum of differential traveltimes should at the least eliminate a source or receiver statics. If only some of the statics delays are eliminated, then this is a partial closure phase; otherwise, it is a total closurephase equation. This will now be demonstrated by examples using closure-phase corrections in traveltime tomography, hypocenter locations, and reflection stacking.

\section{Traveltime tomography with closure phase}

Standard traveltime tomography (Nolet, 1987) is a method for finding the slowness model $\mathbf{s}$ that minimizes the sum of the squared traveltime residuals:

$$
\epsilon=\frac{1}{2} \sum_{i, k} \overbrace{\left(T_{i k}^{\text {syn }}-T_{i k}^{\text {obs }}\right)^{2}}^{\text {traveltime residual }}+\frac{\lambda}{2}\|\mathbf{D s}\|^{2},
$$

where $\lambda$ is a positive damping scalar; obs and syn denote the observed and synthetic traveltimes, respectively; and $\mathbf{D}$ is an operator suited to the type of regularization method. In equation 5 , the traveltime symbols transform as $\bar{T}_{\alpha_{p}} \rightarrow T_{i k}^{\mathrm{syn}}$ and $T_{\beta_{p}} \rightarrow T_{i k}^{\mathrm{obs}}$.

The slowness model is discretized into a grid of $N$ unknown values of slowness and represented by the $N \times 1$ vector $\mathbf{s}$. The traveltimes can be for transmission, refraction, or reflection events, or any combination of these data.

Typically, an iterative gradient method is used to determine the optimal slowness model, where the misfit gradient is given by

$$
\begin{aligned}
\frac{\partial \epsilon}{\partial s_{k^{\prime}}} & =\sum_{i, k}\left(T_{i k}^{\mathrm{syn}}-T_{i k}^{\mathrm{obs}}\right) \frac{\partial T_{i k}^{\mathrm{syn}}}{\partial s_{k^{\prime}}}+\lambda a_{k^{\prime}}, \\
& =\sum_{i, k}\left(T_{i k}^{\mathrm{syn}}-T_{i k}^{\mathrm{obs}}\right) L_{i k}^{k^{\prime}}+\lambda a_{k^{\prime}}
\end{aligned}
$$

Figure 3. Rays for (a) standard, (b) partial closure phase, and (c) total closure phase traveltime tomography. The velocity is updated by smearing the single residual into slowness cells visited by the illustrated rays.

\section{b) Tomography with partial closure phase}

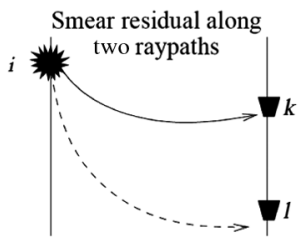

$\Delta \Phi_{i k l}=T_{i k}^{\mathrm{obs}}-T_{i k}^{\mathrm{syn}}$

- ( $\left.T_{i l}^{\mathrm{obs}}-T_{i l}^{\mathrm{syn}}\right)$ c)

Tomography with total closure phase

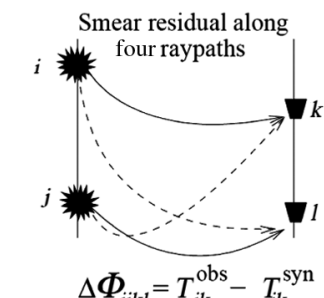

$-\left(T_{i l}^{\text {obs }}-T_{i l}^{\text {syn }}\right)-\left(T_{j k}^{\text {obs }}-T_{j k}^{\text {syn }}\right)$

$$
+\left(T_{j 1}^{\mathrm{obs}}-T_{j 1}^{\mathrm{syn}}\right)
$$

\section{Absolute time $=$ Global velocity anomalies}

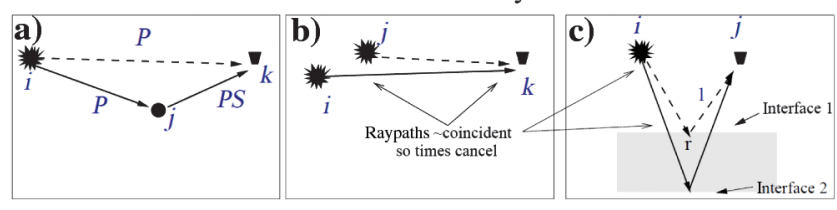

Differential time $=$ Loacl velocity anomalies

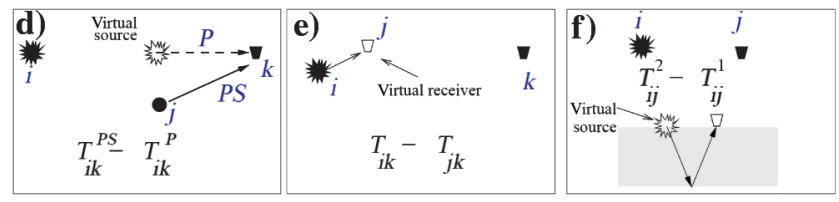

Figure 4. Imaging associated with (a-c) absolute traveltimes tends to globally update the model between the actual sources and receivers, whereas target-oriented imaging of (d-f) differential traveltimes tends to locally update the model between the virtual sources and receivers. The examples are for, from left to right, PS transmission data, PP transmission data, and reflection data. 
where

$$
a_{k^{\prime}} \stackrel{\text { def }}{=}\left(\mathbf{D}^{T} \mathbf{D} \mathbf{s}\right)_{k^{\prime}}
$$

and $L_{i k}^{k^{\prime}}$ is the raypath segment length in the $k^{\prime}$ slowness cell associated with the ray that starts at the $i$ th source and ends at the $k$ th receiver. For the single ray shown in Figure 3a, the slowness is updated by smearing the weighted traveltime residual $L_{i k}^{k^{\prime}} \Delta T_{i k}$ into the $k^{\prime}$ cells visited by the $i k$ ray.

To overcome the problems with source and receiver statics, Zhou and Schuster (2000) propose ITT for seismic data; for earthquake applications, a related tomography method is independently introduced by Zhang and Thurber (2003) except hypocenters are inverted for as well. ITT differs from standard traveltime tomography (Figure 3a) in that the traveltime residuals in equation 6 are replaced by the closure-phase residuals $\Delta \Phi_{i j k l}$ associated with equation 3 or 4 . For the four-point closure-phase residual $\Delta \Phi_{i j k l}$ we have

$$
\Delta \Phi_{i j k l}=\Phi_{i j k l}^{\mathrm{syn}}-\Phi_{i j k l}^{\mathrm{obs}}
$$

and the associated misfit function is

$$
\epsilon=\frac{1}{2} \sum_{B} \Delta \Phi_{i j k l}^{2}+\frac{\lambda}{2}\|\mathbf{D s}\|^{2},
$$

where $B$ is the set of source and receiver pairs corresponding to the differential traveltimes. Note that the receiver and source statics errors in $\Delta \Phi_{i j k l}$ are eliminated because the subtracted traveltimes honor the closure-phase formula in equation 3 .

The formula for the $k^{\prime}$ th component of the misfit gradient vector is given by

$$
\begin{aligned}
\frac{\partial \epsilon}{\partial s_{k^{\prime}}} & =\sum_{B} \Delta \Phi_{i j k l} \frac{\partial \Phi_{i j k l}^{\mathrm{syn}}}{\partial s_{k^{\prime}}}+\lambda a_{k^{\prime}} \\
& =\sum_{B} \Delta \Phi_{i j k l}\left[\frac{\partial T_{i k}^{\mathrm{syn}}}{\partial s_{k^{\prime}}}-\frac{\partial T_{i l}^{\mathrm{syn}}}{\partial s_{k^{\prime}}}+\frac{\partial T_{j l}^{\mathrm{syn}}}{\partial s_{k^{\prime}}}-\frac{\partial T_{j k}^{\mathrm{syn}}}{\partial s_{k^{\prime}}}\right]+\lambda a_{k^{\prime}} \\
& =\sum_{B} \Delta \Phi_{i j k l}\left[L_{i k}^{\left(k^{\prime}\right)}-L_{i l}^{\left(k^{\prime}\right)}+L_{j l}^{\left(k^{\prime}\right)}-L_{j k}^{\left(k^{\prime}\right)}\right]+\lambda a_{k^{\prime}} .
\end{aligned}
$$

For a single-closure residual, the interpretation of this formula is that the closure residual $\Delta \Phi_{i j k l}$ is weighted by the associated raypath segment lengths and smeared over the cells visited by the four rays indexed by $i k, i l, j k$, and $j l$ (see Figure $3 \mathrm{c}$ ).

Smearing one residual over so many rays will lead to a loss of spatial resolution (Zhou and Schuster, 2000). A partial remedy is to use the three-point closure-phase residual in equation 4 so that smearing of a residual is only over the two rays shown in Figure 3b, but now there are nonzero receiver statics. Another partial remedy is to design the dashed-solid ray pairs so that the solid ray is partnered with a nearly coincident negative ray outside the region of interest, as discussed below.

\section{Localization of velocity anomalies.}

Imaging of differential traveltimes can be designed so that the velocities are mostly updated locally around the virtual sources and receivers, as illustrated in Figure 4. For example, a single traveltime residual for any of the experiments in Figures $4 a-4 c$ is smeared over the entire raypath between the actual source and receiver. If the dashed (with a negative residual) and solid (with a positive residual) ray pairs are nearly coincident, then the slowness update from a single residual will be nearly zero along these nearly coincident paths (the length of the ray segment in a slowness pixel will be effectively the same for nearly coincident solid and dashed rays, so the weighted positive and negative residuals add to zero in this pixel for the slowness update). Figure $4 \mathrm{c}$ shows that the dashed-solid ir and $j r$ rays in the CMP gather share nearly the same raypaths in the uninteresting top layer, so the slowness update there is mostly negligible. Most of the slowness update is in the bottom layer of Figure $4 \mathrm{f}$ where there are no dashed rays. This update-localization property is also demonstrated with the DD method for estimation of hypocenters (Waldhauser and Ellsworth, 2000; Zhang and Thurber, 2003; Michelini and Lomax, 2004), reduced time migration (Sheley and Schuster, 2003; Zhou et al., 2006), and ITT (Hanafy and Schuster, 2007).

\section{Synthetic data test}

The ITT algorithm is tested on synthetic refraction data (Zhou and Schuster, 2000) associated with the three-layer velocity model in Figure 5a. First-arrival traveltimes for sources and receivers on the surface are computed by a finite-difference solution to the eikonal equation, and the tomographic method described in Nemeth et al. (1997) is used for the inversion. In this example, the threepoint closure-phase equation 4 is used for the ITT residual so that the source statics in any shot gather is eliminated. The first-arrival traveltime for any trace in a shot gather is reduced by that of the trace closest to the source; we call this nearest-offset trace the master trace. The nearest-offset trace is selected to maximize difference in the raypath lengths of the ray pair, which reduces the loss of spatial resolution (see Appendix A).

The standard traveltime tomogram is shown in Figure 5b, and it represents the calibration tomogram computed for data without a source static. When the observed traveltimes are increased by $15 \mathrm{~ms}$ due to a uniform source static, standard traveltime tomography can no longer provide correct information about the shallow velocity structure as shown in Figure 5c, whereas the ITT tomogram in Figure 5d reconstructs the velocity distribution quite well. The low-wavenumber component of the slowness field is almost recovered due to the nature of the acquisition geometry and the near-offset selection of the master trace. The rays for the master traces are mostly along the surface, and the surface velocity is not very far away from the one provided by the initial model. In fact, the surface velocity is much easier to estimate than the deeper one and is the reason why the ITT method can work well with a surface-refraction experiment.

\section{Hypocenter estimation with closure phase}

Earthquake seismologists use the DD method to improve the accuracy of estimating hypocenters of earthquakes (Waldhauser and Ellsworth, 2000) and tomographic velocities (Zhang and Thurber, 2003). They use the three-point closure-phase formula given in equation 4 . The DD residual $\Delta \Phi_{i j k}=\Phi_{i j k}^{\mathrm{syn}}-\Phi_{i j k}^{\mathrm{obs}}$ is linearized with respect to the coordinate perturbations of the $i$ th and the $j$ th seismic sources, i.e., $\left(\Delta x^{i}, \Delta y^{i}, \Delta z^{i}\right)$ and $\left(\Delta x^{j}, \Delta y^{j}, \Delta z^{j}\right)$, respectively. This 
residual is also linearized with respect to the differential traveltimes $\Delta \tau^{i}$ and $\Delta \tau^{j}$ for the two nearby earthquakes to give

$$
\begin{aligned}
\Phi_{i j k} & \approx \frac{\partial T_{i k}}{\delta x^{i}} \Delta x^{i}+\frac{\partial T_{i k}}{\delta y^{i}} \Delta y^{i}+\frac{\partial T_{i k}}{\delta z^{i}} \Delta z^{i}+\Delta \tau^{i} \\
& -\frac{\partial T_{j k}}{\delta x^{j}} \Delta x^{j}-\frac{\partial T_{j k}}{\delta y^{j}} \Delta y^{j}-\frac{\partial T_{j k}}{\delta z^{j}} \Delta z^{j}-\Delta \tau^{j}
\end{aligned}
$$

compared well against the traditional statics methods (Sheng et al., 2005).

In this method, let $T_{i k}$ denote the picked reflection traveltime associated with the $i$ th source and $k$ th receiver and let the tilde symbol $\sim$ indicate the removal of the reflection traveltime moveout. This moveout can be estimated by a spatial smoothing, for example, a cubic smoothing spline (Eubank, 1999, chapter 5), of the picked $T_{i k} \mathrm{~s}$ within a gather of interest. In a common-source gather (CSG),
It can be said that "...we avoid the need for station receiver corrections or high-accuracy of predicted traveltimes for the portion of the raypath that lies outside the focal volume. This approach is especially useful in regions with a dense distribution of seismicity, i.e., where distances between neighboring events are only a few kilometers or less" (Waldhauser, 2001).

Waldhauser and Ellsworth (2000) apply the DD hypocenter method to earthquake data originating along California's Hayward fault, and the result is shown in Figure 6. Here, the hypocenter locations computed by the DD method in Figure $6 \mathrm{~b}$ are much more focused along the fault than the Figure 6a hypocenters estimated by the standard location algorithm. In this case, the traveltimes are those of S-wave arrivals.

An extension of the DD method was developed by Zhang and Thurber (2003) who estimate not just "the relative event locations, but also their absolute locations and velocity structure." They demonstrate with synthetic data that better DD estimates of the hypocenters lead to more accurate velocity tomograms, and vice versa. For the DD method, it can be shown that the differential traveltime of two proximal earthquakes is mostly a result of the local velocity error around that region. This point is illustrated in Figure $4 \mathrm{a}$ and $4 \mathrm{~d}$.

\section{Reflection statics with closure phase}

Reflection arrivals in a land survey typically suffer from time delays due to, for example, near-surface velocity variations at the source and the receiver. These unwanted time delays must be corrected to achieve stacked sections with high S/Ns. A typical procedure for iteratively correcting these statics is to apply an NMO operation to a CMP, correlate a pilot trace (initialized by stacking the NMO-corrected traces, for example) with traces in the NMO-corrected gather to obtain the corrective time shifts, and use a least-squares method (Wiggins et al., 1976; Docherty, 1992; Marsden, 1993a, 1993b, 1993c; Cox, 1999) to invert for the static corrections. The pilot trace is updated by stacking, and this procedure is iteratively applied until the statics flatten the specified reflections in the NMO-corrected gather. It is effectively used with land seismic data but can require a computationally costly inversion procedure. To avoid this cost, Yu (2000) and Sheng et al. (2005) present a closure-phase statics-correction method to directly estimate the source and receiver statics without solving a large system of equations. Results with field data b)

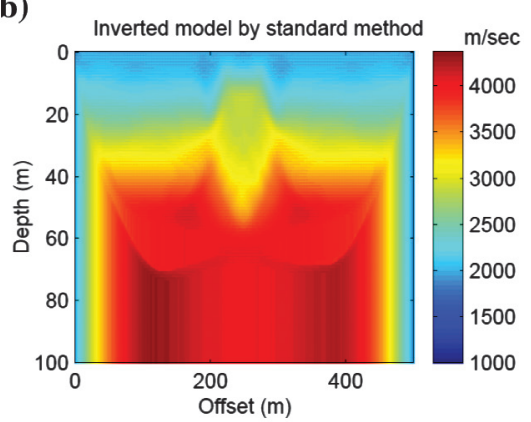

d)

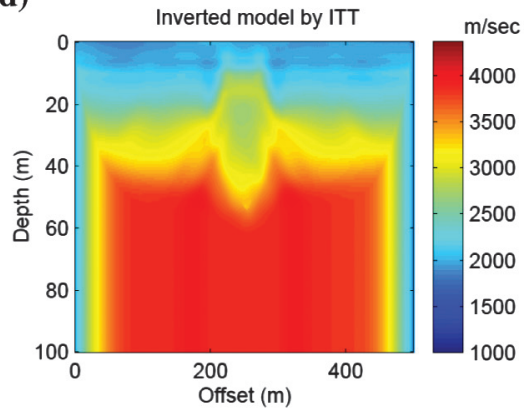

Figure 5. Synthetic model and inversion results (Zhou, 1999) for surface refraction exPeriment: The shot and receiver interval is $2 \mathrm{~m}$, and the grid size is $0.5 \mathrm{~m} \times 0.5 \mathrm{~m}$ Velocities are $2000 \mathrm{~m} / \mathrm{s}$ (upper layer), $3000 \mathrm{~m} / \mathrm{s}$ (middle layer), and $4000 \mathrm{~m} / \mathrm{s}$ (lower layer). (a) True model, (b) inverted model from standard traveltime tomography with

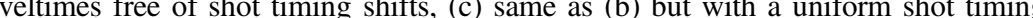
with this $15-\mathrm{ms}$ shot-timing shift. A linear-gradient velocity model is used for the initial model with a surface velocity of $1000 \mathrm{~m} / \mathrm{s}$ and a deep velocity of $5000 \mathrm{~m} / \mathrm{s}$.
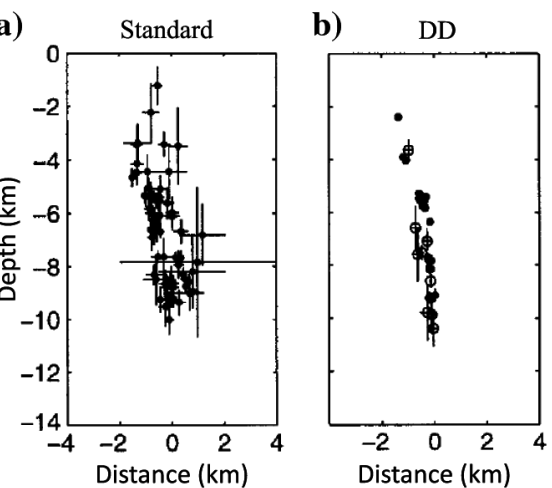

Figure 6. Hypocenter locations estimated by the (a) standard and (b) DD methods (adapted from Waldhauser and Ellsworth, 2000). The input traveltimes are those for S-wave arrivals, and the vertical slices are along similar positions across the Hayward fault. 
the three-point partial closure phase, similar to equation 4 except that the source statics are eliminated, is applied to the $\tilde{T}_{i k}$ s to yield

$$
\begin{aligned}
\tilde{\Phi}_{i k l} & =\tilde{T}_{i k}-\tilde{T}_{i l}, \\
& =\tilde{t}_{i k}-\tilde{t}_{i l}+\tau_{k}^{\mathrm{rec}}-\tau_{l}^{\mathrm{rec}}, \\
& =\tau_{k}^{\mathrm{rec}}-\tau_{l}^{\mathrm{rec}}
\end{aligned}
$$

where the actual traveltimes $\tilde{t}_{i k}$ and $\tilde{t}_{i l}$ after moveout correction cancel one another so that $\tilde{\Phi}_{i k l}$ is the difference between the $k$ th and $l$ th receiver statics.

Equation 12 can be solved for the $k$ th receiver statics as

$$
\tau_{k}^{\mathrm{rec}}=\tau_{l}^{\mathrm{rec}}+\tilde{\Phi}_{i k l}
$$

and it is valid for a range of $l$ and $i$ values. For example, setting $l=k-1$, and successive substitution in the above equation gives

$$
\begin{aligned}
\tau_{k}^{\mathrm{rec}} & =\tau_{k-1}^{\mathrm{rec}}+\tilde{\Phi}_{i k k-1}, \\
& =\tau_{k-2}^{\mathrm{rec}}+\tilde{\Phi}_{i k-1 k-2}+\tilde{\Phi}_{i k k-1}, \\
& =\tau_{k-3}^{\mathrm{rec}}+\tilde{\Phi}_{i k-2 k-3}+\tilde{\Phi}_{i k-1 k-2}+\tilde{\Phi}_{i k k-1}, \\
& \vdots \\
& =\tau_{0}^{\mathrm{rec}}+\sum_{l=1}^{k} \tilde{\Phi}_{i l l-1} .
\end{aligned}
$$

a)

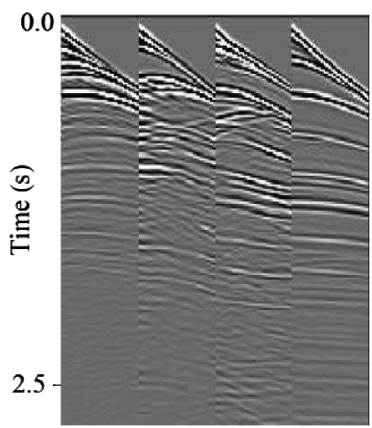

b) Shot gathers+statics

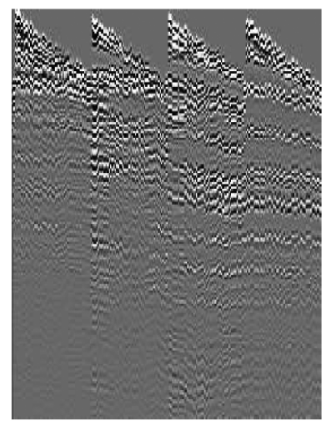

d)

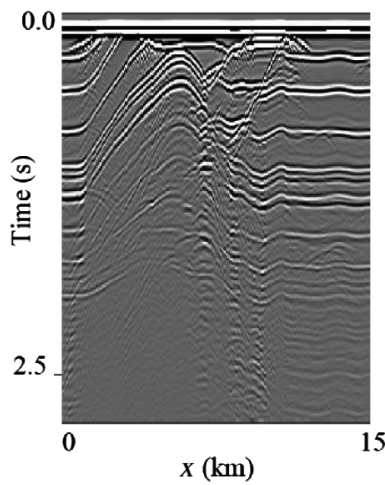

e) Stacked section+statics

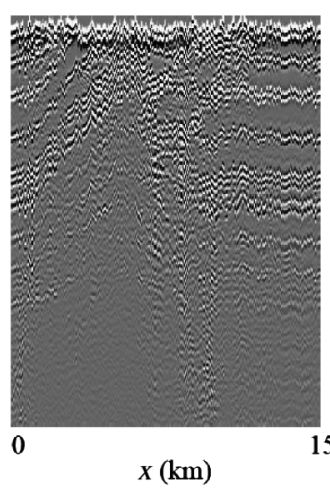

Figure 7. Shot gathers for the SEG/EAGE model with (a) no statics, (b) source and receiver statics, and (c) statics corrected by the closure-phase equation 16 . The corresponding stacked sections are shown in (d-f). Illustration is adapted from Yu (2000).
The left side is independent of $i$, so averaging over $i$ yields the final formula for the receiver statics:

$$
\tau_{k}^{\mathrm{rec}}=\tau_{0}^{\mathrm{rec}}+\frac{1}{N_{\mathrm{csg}}} \sum_{i=1}^{N_{\mathrm{csg}}} \sum_{l=1}^{k} \tilde{\Phi}_{i l l-1},
$$

where $N_{\text {csg }}$ is the number of CSGs over which the averaging is carried out.

On the other hand, in a common receiver gather (CRG), equation 4 can be applied to give

$$
\begin{aligned}
\tilde{\Theta}_{i j k} & =\tilde{T}_{i k}-\tilde{T}_{j k}, \\
& =\tilde{t}_{i k}-\tilde{t}_{j k}+\tau_{i}^{\mathrm{src}}-\tau_{j}^{\mathrm{src}}, \\
& =\tau_{i}^{\mathrm{src}}-\tau_{j}^{\mathrm{src}}
\end{aligned}
$$

where the moveout correction in the CRG renders the traveltimes $\tilde{t}_{i k}$ and $\tilde{t}_{j k}$ to be equal to each other. Following the derivation from equations $14-16$, we obtain

$$
\begin{aligned}
\tau_{i}^{\mathrm{src}} & =\tau_{j}^{\mathrm{src}}+\tilde{\Theta}_{i j k}, \\
& =\tau_{0}^{\mathrm{src}}+\sum_{l=1}^{i} \tilde{\Theta}_{l l-1 k}, \\
& =\tau_{0}^{\mathrm{src}}+\frac{1}{N_{c r g}} \sum_{k=1}^{N_{c r g}} \sum_{l=1}^{i} \tilde{\Theta}_{l l-1 k},
\end{aligned}
$$

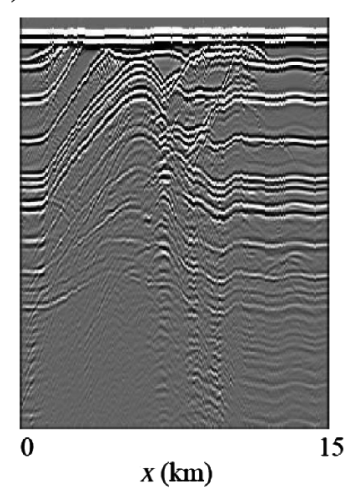

c) Corrected shot gathers

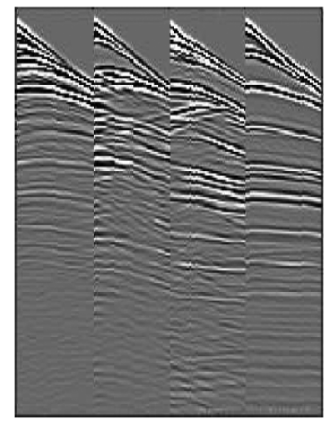

f) Corrected stacked section where $N_{\text {crg }}$ is the number of CRGs over which the averaging is carried out.

These formulas can be used for refraction statics (Palmer, 1981) if the reflection traveltime moveout is replaced by a linear moveout operation to flatten the first arrivals, which, in practice, are more easily picked and are less influenced by the deeper medium. However, the apparent statics might depend on the depth of the reflector (section 6.2.1 in Cox, 1999).

\section{Synthetic data test}

A total of 312 acoustic shot gathers (four are shown in Figure 7a) were computed for the SEG/ EAGE overthrust model, where each shot gather contains 120 traces. Each trace has a recording length of $2.8 \mathrm{~s}$ with a sample interval of $8 \mathrm{~ms}$. The source and receiver intervals are 50 and $25 \mathrm{~m}$, respectively, and the source-receiver offsets range from 50 to $3280 \mathrm{~m}$. Random statics at shot and receiver stations are applied to the traces with a maximum statics value of $48 \mathrm{~ms}$. Four distorted shot gathers are shown in Figure 7b. In this example, the traveltimes of the first-arrival refractions are inserted into equation 14 to iteratively estimate the receiver static shifts and into equation 16 to iteratively estimate the source statics. Each iteration consists of applying the optimal linear moveout to the traces that best flattens the first arrivals, using the closure-phase equa- 
tion to estimate statics, and removing these static shifts to correct the data. These corrected shot gathers are used as input data for the next iteration. Three such iterations are carried out.

Figure 7c shows several windowed shot gathers after applying static correction to the Figure $7 \mathrm{~b}$ data. It is clear that the corrected shot gathers mostly correct the statics and resemble the statics-free shot gathers shown in Figure 7a. Also, the final stacked section shown in Figure $7 \mathrm{f}$ resembles the statics-free section shown in Figure $7 d$.

\section{STATIONARY CLOSURE PHASE}

The closure-phase equation that satisfies a zero stationarity condition is defined as

$$
\operatorname{extrm}_{\gamma}\left[\sum_{p=1}^{P}\left(\bar{T}_{\alpha_{p}}-T_{\beta_{p}}\right)\right]=0
$$

where extrm $r$ denotes the extremum value of the bracketed term with respect to variations of the index denoted by $\gamma$. This extremum point, also known as a stationary point, is denoted as $\gamma^{*}$. As will be shown, the stationary closure-phase principle is equivalent to Fermat's principle that can be used to (1) predict long-offset traveltimes from shorter offset traveltimes, (2) check for accuracy in traveltime picks, (3) predict short-offset traveltimes from long-offset traveltimes, and (4) eliminate source and receiver statics for traveltime tomography, earthquake location, migration, and waveform inversion.

\section{Stationary closure phase for reflections}

Consider the surface seismic profile (SSP) geometry in Figure 8a characterized by primary reflection rays from the shallow and deep interfaces. The goal is to redatum the surface source and receivers to the shallow interface so that, with virtual sources (see Figure 8c) and receivers closer to the target, a more accurate velocity image of the middle layer can be computed.

Assume that the traveltimes of the primary reflections from the deeper interface are picked and denoted by $T_{x B}^{\text {primary }}$, and the reflections from the shallow interface can be used to obtain the traveltime $T_{x A}^{\text {direct }}$ for a direct wave to propagate from $\mathbf{x}$ to the specified position A on the shallow interface (see Schuster [2005b], section "Interferometric CDP traveltime tomography"). The dashed diffraction ray in Figure $8 \mathrm{a}$ is restricted to intersect the source at $\mathbf{x}$ and the specified point $\mathbf{A}$ and also to coincide with the specular reflection ray $A B$. Inserting $p=1, \gamma=x$, and

$$
\begin{gathered}
\bar{T}_{\alpha_{1}} \rightarrow T_{x B}^{\text {diff. }}=T_{A B}^{\text {primary }}+T_{x A}^{\text {direct }}, \\
T_{\beta_{1}} \rightarrow T_{x B}^{\text {primary }},
\end{gathered}
$$

into equation 19 produces, in line with Fermat's interferometric principle,

$$
\begin{aligned}
& \operatorname{extrm}_{x}\left[T_{x B}^{\text {diff. }}-T_{x B}^{\text {primary }}\right] \\
& \quad=\operatorname{extrm}_{x}\left[T_{A B}^{\text {primary }}+T_{x A}^{\text {direct }}-T_{x B}^{\text {primary }}\right]=0,
\end{aligned}
$$

where the reflection traveltime $T_{x B}^{\text {primary }}$ for a source at $\mathbf{x}$ can be used to calculate the virtual reflection traveltime $T_{A B}^{\text {primary }}$ with the virtual source at $\mathbf{A}$ in Figure 8c. Here, the differential time is the difference between the specular reflection traveltime $T_{x B}^{\text {primary }}$ and the diffraction traveltime $T_{x B}^{\text {diff. }}=T_{A B}^{\text {primary }}+T_{x A}^{\text {direct }}$, which by Fermat's principle is always greater than 0 unless the source coincides with the stationary point at $\mathbf{x}^{*}$ in Figure $8 \mathrm{~b}$.

Equation 22 can be rearranged as

$$
\operatorname{extrm}_{x}\left[T_{x B}^{\text {primary }}-T_{x A}^{\text {direct }}\right]=T_{A B}^{\text {primary }},
$$

to give (Schuster, 2005a, 2005b) the virtual reflection time $T_{A B}^{\text {primary }}$ for sources redatumed to the shallow interface. A similar formula can be used to redatum the receiver to the shallowest interface and so get virtual reflection traveltimes from a virtual acquisition geometry on the shallow interface.

As an example, Figure 9 shows a radar velocity model and the associated radar velocity tomograms after inverting reflection traveltimes picked from simulated ground-penetrating radar. There are 70 evenly spaced transmitted locations on the surface, and each transmitter propagates radar waves into 24 receivers at $1-\mathrm{m}$ spacing. The standard velocity tomogram inverted from the reflection traveltimes is on the upper right and does not reveal much of the actual detail seen in the original model. The traveltimes associated with reflections from interfaces $\mathrm{A}$ and $\mathrm{B}$ are picked, and equation 23 is used to redatum the sources and receivers to be at the first reflect-
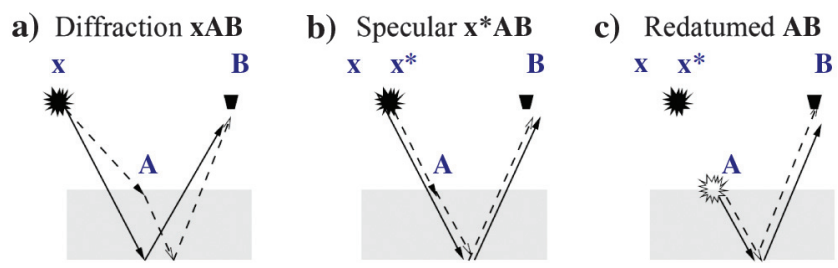

Figure 8. Ray diagram for an SSP experiment in which the source at $\mathbf{X}$ and receiver at $\mathbf{B}$ are on the earth's surface and $\mathbf{A}$ is a specified virtual receiver point on the top interface. The specular reflection is depicted as a solid ray, and the (a) dashed diffraction ray only honors Snell's law if $\mathbf{x}$ is moved to (b) the stationary source point at $\mathbf{x}^{*}$. The diagram in (c) depicts the reflection ray associated with the source redatumed to $\mathbf{A}$.

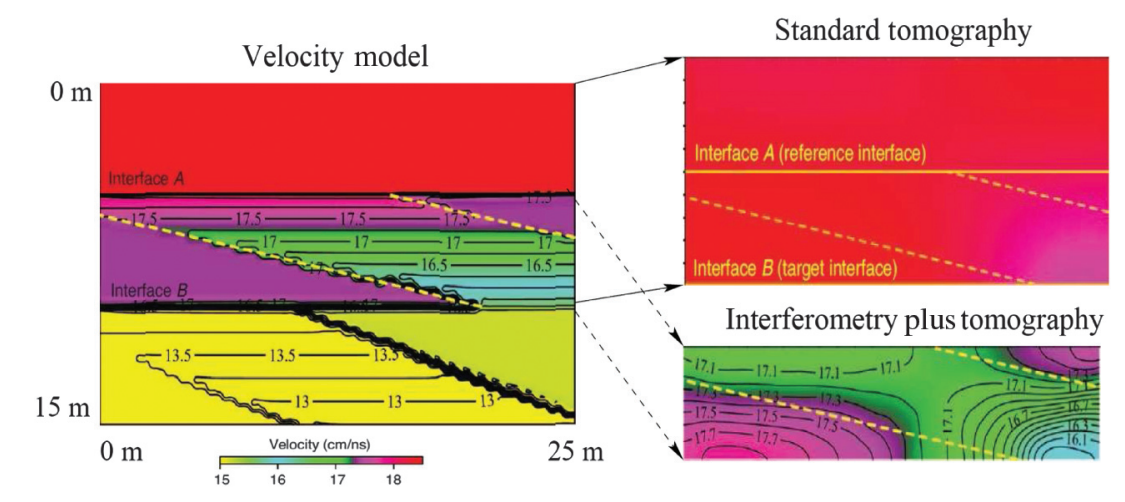

Figure 9. Radar velocity model on the left and (upper right) standard and (lower right) interferometry tomograms. Input data are traveltimes for reflections from interfaces A and $\mathrm{B}$ for shot gathers excited by radar transmitters and recorded by the receivers at $z=0 \mathrm{~m}$. Illustrations are adapted from Hanafy and Schuster (2007). 
ing interface. The corresponding virtual traveltimes are inverted, and the resulting tomogram is shown in the lower right of Figure 9. It reveals much more detail about the dipping layers than is seen in

Four-point stationary closure phase for refractions

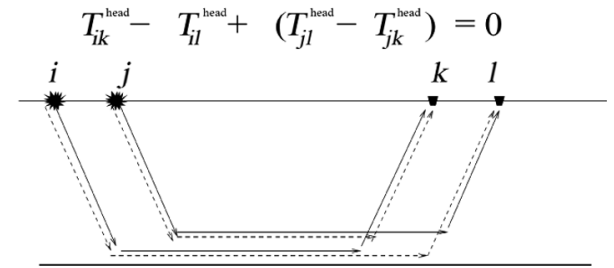

Figure 10. Head-wave raypaths for the four-point stationary closure-phase formula in equation 25 .

Six-Point stationary closure phase for refractions
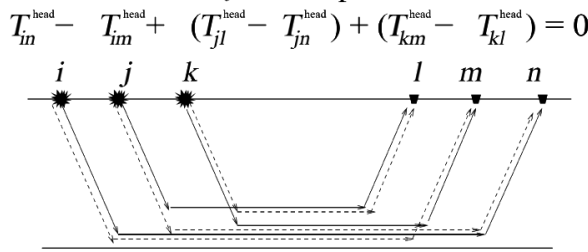

Figure 11. Head-wave raypaths for the six-point stationary closurephase formula in equation 26 . the standard tomogram. Alternatively, a nonstationary approach to traveltime tomography could be used with the closure-phase equations associated with Figure 4.

\section{Stationary closure phase for refractions}

Equation 19 can also be applied to the head-wave arrivals shown in Figure 10 to give Fermat's principle for head waves. Here, we set the $\bar{T}_{\alpha_{p}}$ and $T_{\beta_{p}}$ in equation 19 to be the head-wave traveltimes

$$
T_{\beta_{p}} \rightarrow T_{\beta_{p}}^{\mathrm{head}} ; \bar{T}_{\alpha_{p}} \rightarrow T_{\alpha_{p}}^{\mathrm{head}},
$$

where the head wave is assumed to be from the same refractor for any source-receiver pair and all source-receiver pairs have an offset greater than the critical distance. For head waves, setting $p=2$ in equation 19 and setting $\alpha_{1}=i k, \alpha_{2}=j l, \beta_{1}=i l, \beta_{2}=j k$ gives the four-point closure-phase equation 3 :

$$
T_{i k}^{\text {head }}-T_{i l}^{\text {head }}+T_{j l}^{\text {head }}-T_{j k}^{\text {head }}=0,
$$

which is zero for any source-receiver pair having a critical offset. As shown in Appendix B, equation 25 is the stationary phase condition for the reciprocity equations of correlation and convolution types. It can be generalized to higher order conditions such as the six-point stationary closure-phase equation:

$$
T_{i n}^{\text {head }}-T_{i m}^{\text {head }}+\left(T_{j l}^{\text {head }}-T_{j n}^{\text {head }}\right)+\left(T_{k m}^{\text {head }}-T_{k l}^{\text {head }}\right)=0 \text {, }
$$

a)

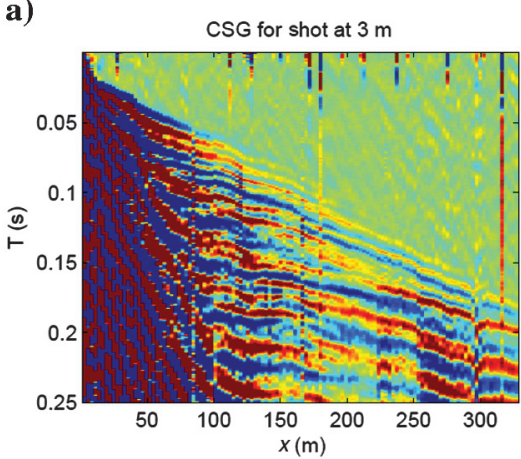

b)

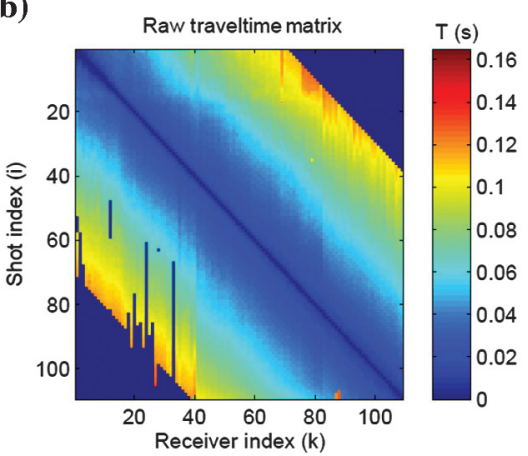

c)

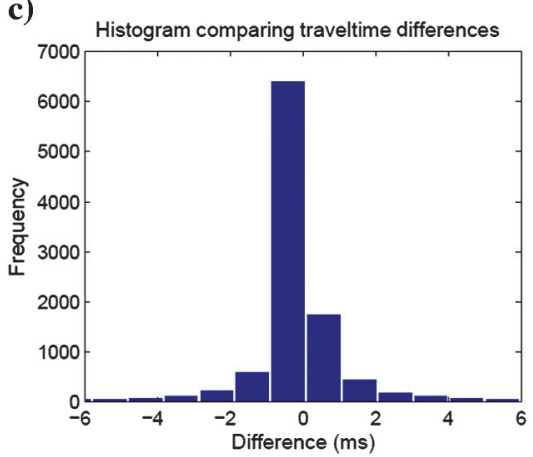

d)

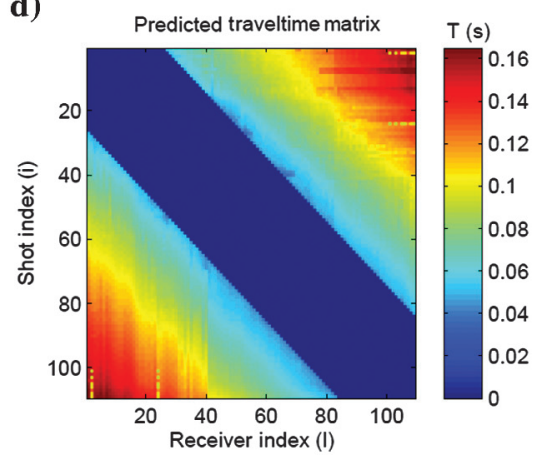

Figure 12. A typical (a) shot gather recorded over the Qadimah fault in Saudi Arabia, and (b) first-arrival traveltimes picked from the Qadimah data as a function of the shot and receiver indices. The predicted traveltimes using equation 25 (with the Qadimah short-offset traveltimes as input) are displayed in (c). In this case, the gaps in the Figure $12 \mathrm{~b}$ matrix are filled by inserting traveltimes from shorter offset events into equation 25 to obtain the longer offset events. Panel (d) reveals that the predicted traveltimes agree with the actual ones to less than $T / 4=6 \mathrm{~ms}$.

and the associated raypaths are shown in Figure 11 . This equation is a special case of the general closure-phase equation 19 for an arbitrary number of points.

Equation 25 has at least four practical uses:

1) It is a general reciprocity relation that can be used to check the accuracy of picked traveltimes of head waves. For example, assume all four of the traveltimes in equation 25 are picked with an undetermined picking error. Inserting these picked times into this equation should equal zero within an acceptable tolerance of one-fourth the dominant period $T$ of the source wavelet. If this condition is not satisfied, the associated events should be picked over again for better accuracy. For head waves, equation 25 is a reciprocity principle that complements the standard one where violations of the rule $T_{i j}=T_{j i}$ are used to identify mispicked traveltimes.

2) Equation 25 can be used to predict unpickable traveltimes of long-offset head waves from shorter-offset refraction traveltimes. As an example, Figure $12 \mathrm{~b}$ presents the traveltime matrix associated with picked traveltimes for different source-receiver pairs; a typical shot gather from which these traveltimes were picked is shown in Figure 12a. The gaps in the Figure 12b matrix are due to the noisy traces in which the first-arrival traveltimes could not be accurately picked. 
Equation 25 can be arranged as

$$
T_{i l}^{\mathrm{head}}=T_{i k}^{\mathrm{head}}+T_{j l}^{\mathrm{head}}-T_{j k}^{\mathrm{head}},
$$

to compute the long-offset traveltime $T_{i l}^{\text {head }}$ in shot gathers such as Figure 10 from the shorter offset traveltimes. Thus, the gaps in the original traveltime matrix shown in Figure 12b are filled to give Figure $12 \mathrm{c}$. To estimate the reliability of the predicted traveltimes, Figure 12d depicts the histogram of the traveltime differences between the picked and the predicted traveltimes, where the picked traveltimes are from the traces with a high $\mathrm{S} / \mathrm{N}$. As shown, the traveltime residuals are far less than the acceptable tolerance of $T / 4$. Statistical measures of picking errors such as the mean traveltime at a geophone and the standard deviation of picking error can now be readily estimated with the traveltime predicted from equation 27.

3) Equation 25 can be used to determine if a first arrival is a head wave from the same refractor or a diving wave. If equation 25 is equal to zero (within the specified tolerance) for the picked traveltimes, then the picked events are associated with a head-wave refractor. Otherwise, they could be purely diving waves or the traveltimes are picked for head waves from different refractors. In either case, this is useful diagnostic information.

4) The traveltime prediction equation 27 is the basis for generating far-offset refraction traces. Let two refraction arrivals $u(t)$ and $v(t)$ be approximated in the Fourier domain by $U(\omega)=$ $A(\omega) \exp \left(i \omega \tau_{u}\right)$ and $V(\omega)=B(\omega) \exp \left(i \omega \tau_{v}\right)$, respectively, where the $\tau$ s denote the traveltimes. Then $U(\omega) V(\omega)=$ $A(\omega) B(\omega) \exp \left(i \omega\left[\tau_{u}+\tau_{v}\right]\right)$, and $U^{*}(\omega) V(\omega)=A^{*}(\omega) B(\omega)$ $\exp \left(i \omega\left[-\tau_{u}+\tau_{v}\right]\right)$. In the time domain, the former and the latter quantities correspond to $u(t) * v(t)$ and $u(t) \star v(t)$, respectively, where $*$ denotes convolution, and $\star$ denotes crosscorrelation. The exponential arguments are interpreted as saying that traveltime addition corresponds to convolution, whereas traveltime subtraction corresponds to crosscorrelation of the product functions in the time domain. Therefore, the traveltime algebra in equation 27 implies how a far-offset (indexed by $i l$ ) refraction trace can be predicted by a combination of shorter offset (indexed by $i k, j l$, and $j k$ ) refraction traces. Repeat this procedure over $N$ different combinations of shorter-offset traces to predict $N$ versions of the far-offset traces at the same offset position. These $N$ versions of predicted traces can be stacked together to enhance the $\mathrm{S} / \mathrm{N}$ by $\sqrt{N}$ of this far-offset supervirtual trace (Mallinson et al., 2011; Bharadwaj et al., 2013).

Stationary closure phase for diffractions

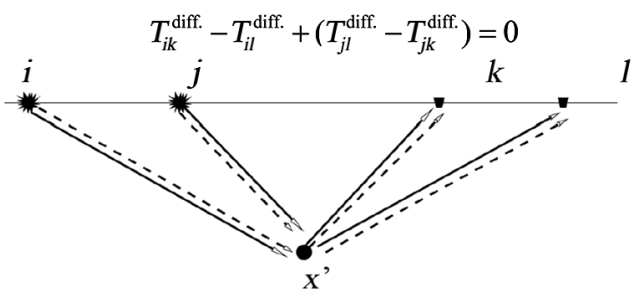

Figure 13. Four-point stationary closure-phase equation and rays for a diffraction point at $\mathbf{x}^{\prime}$. The stationary equation 27 is valid for any location of the source and receiver locations.

\section{Stationary closure phase for diffractions}

The ray diagrams for the four-point stationary closure-phase principle associated with diffractions from the point scatterer at $\mathbf{x}^{\prime}$ are shown in Figure 13, and the associated stationary closure-phase equation,

$$
T_{i k}^{\text {diff. }}-T_{i l}^{\text {diff. }}+T_{j l}^{\text {diff. }}-T_{j k}^{\text {diff. }}=0
$$

is valid for any location of sources and receivers. This compares with the stationary closure-phase equation 27 for head waves that is valid only if the head-wave traveltimes are from the same refractor and recorded beyond the critical offset.

A practical use of this stationarity condition for diffractions is similar to that for head waves; i.e., it can be used to reinforce the diffraction arrivals by correlation of traces summed over different shotpoints to create virtual diffraction traces, followed by convolution of these virtual traces with the actual traces and summation over different receiver locations (Dai et al., 2011). The end result is an $\mathrm{S} / \mathrm{N}$ enhancement of $\sqrt{N}$, where $N$ is the number of geophones that coincide with the shot locations. Reinforcing the diffractions at the expense of other signals allows for the extraction of diffraction arrivals, which can be useful because they can enhance imaging resolution (Kaschwich et al., 2011; Nikitchenko et al., 2011; Tsingas et al., 2012).

An example of extracting diffractions using the above procedure is shown in Figure 14. Here, shot gathers with random statics shifts are generated by a finite-difference solution to the $2 \mathrm{D}$ acoustic wave equation for the Figure 14a fault model (Dai et al., 2011). The red dashed line in Figure 14a indicates the diffractions from this fault, and a magnified view is shown in Figure 14b. A local median filter
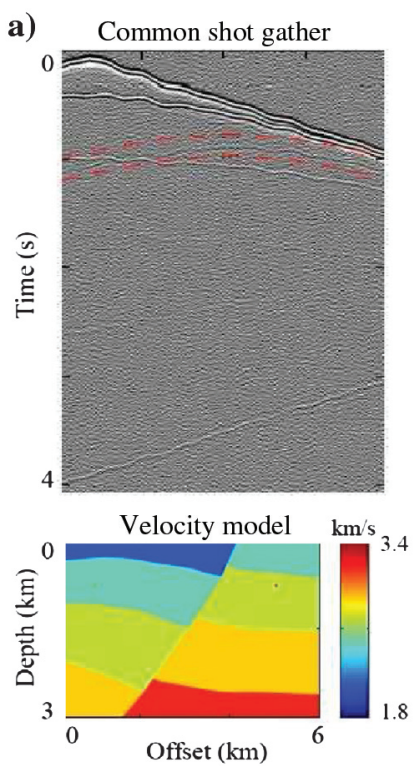

b)

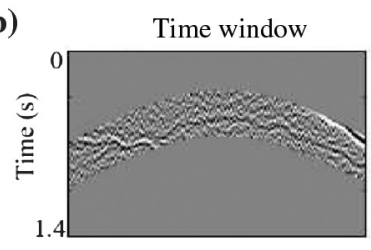

c)

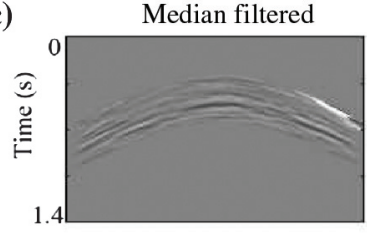

d)

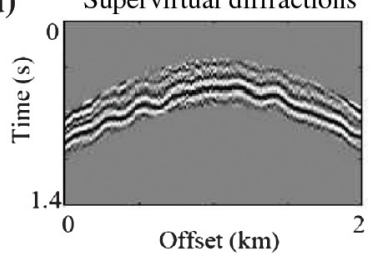

Figure 14. Synthetic data for a fault model. (a) A common shot gather with a source at offset $36 \mathrm{~m}$. Red lines indicate the time window of the diffraction event. (b) The diffraction event within a small time window. (c) The result after median filtering and (d) after processing the raw data to obtain the supervirtual diffraction arrivals. Illustrations are adapted from Dai et al. (2011). 
directed along the major dip of the diffraction curve is used to extract the diffractions, and the result is shown in Figure 14c, where the high-wavenumber features are smoothed out. In comparison, the supervirtual diffractions obtained from 400 shot gathers are shown in Figure $14 d$ where the high-wavenumber features of the actual diffraction curve are retained.

Equation 28 is also valid for surface waves with sources and receivers along the surface. This can easily be shown by moving the diffractor point to the surface in Figure 13 so that it is somewhere between the $j$ and $k$ positions; in this case, the raypaths now resemble those of direct waves or surface waves. Similar to that for head waves and diffraction arrivals, correlation of traces with surface-wave arrivals and summation over different sources reinforces the $\mathrm{S} / \mathrm{N}$ of the surface waves so they can be extracted as signal (Derode et al., 2003; Gerstoft et al., 2006) or filtered as noise (Halliday et al., 2007; Xue et al., 2009).

\section{Stationary closure phase and migration}

The stationary closure-phase equation can also be used to reduce migration defocusing caused by source statics and velocity errors. As an example, consider the migration of transmitted PS-waves to image the location of the orebody shown by the dashed lines in Figure 15. Here, it is assumed that the source at $\mathbf{s}$ along the right well generates a P-wave that converts to a PS transmission wave at the boundary point $\mathbf{x}_{0}$ of the orebody. The resulting transmitted PSwave is recorded at $\mathbf{g}$ along the left well with the traveltime of $\tau_{s x_{0}}^{\mathrm{P}}+\tau_{x_{0} g}^{\mathrm{S}}$. In addition, the transmitted $\mathrm{P}$-wave is also recorded at $\mathbf{g}$ and the traveltime is denoted as $\tau_{s g}^{\mathrm{P}}$. To eliminate the source statics, the trace in the frequency domain $d(\mathbf{s}, \mathbf{g})=$ $A(\mathbf{s}, \mathbf{g}) \exp \left(i \omega\left[\tau_{s x_{0}}^{P}+\tau_{x_{0} g}^{S}\right]\right)$ can be shifted in time by the picked direct-arrival time $\tau_{s g}^{\mathrm{P}}$ to give the shifted trace $d(\mathbf{s}, \mathbf{g})^{\prime}=d(\mathbf{s}, \mathbf{g})$ $\exp \left(-i \omega \tau_{s g}^{\mathrm{P}}\right)=A(\mathbf{s}, \mathbf{g}) \exp \left(i \omega \tau_{s x_{0} g}^{\mathrm{redu}}\right) ;$ here, $\tau_{s x_{0} g}^{\mathrm{redu}}=\tau_{s x_{0}}^{P}+\tau_{x_{0} g}^{S}-\tau_{s g}^{\mathrm{P}}$ is the reduced traveltime, $\omega$ is the angular frequency, and $A(\mathbf{s}, \mathbf{g})$ accounts for geometrical spreading and source wavelet effects. These shifted traces can then be migrated by the diffraction-stack equation for PS migration (Keho, 1986; Sheley, 2001; Luo et al., 2006):

$$
\begin{aligned}
& m(\mathbf{x})=\sum_{\omega, s, g} d(\mathbf{s}, \mathbf{g})^{\prime} \exp \left(-i \omega \tilde{\tau}_{s x g}^{\mathrm{redu}}\right), \\
& =\sum_{\omega, s, g} A(\mathbf{s}, \mathbf{g}) \exp \left(i \omega\left[\tau_{s x_{0} g}^{\mathrm{redu}}-\tilde{\tau}_{s x g}^{\mathrm{redu}}\right]\right),
\end{aligned}
$$

where $\tilde{\tau}_{s x g}^{\mathrm{redu}}=\tilde{\tau}_{s x}^{P}+\tilde{\tau}_{x g}^{S}-\tilde{\tau}_{s g}^{\mathrm{P}}$ denotes the reduced traveltime, whose terms are calculated from an assumed velocity model for a source at $\mathbf{x}$ and a receiver at $\mathbf{y}$; here, $m(\mathbf{x})$ is the transmissivity image $m(\mathbf{x})$ of the orebody boundary. Migrating data with the above equation is denoted as reduced-time migration by Sheley and Schuster (2003)

Assume that the trial image point $\mathbf{x}$ in equation 30 coincides with the boundary point $\mathbf{x}_{0}$ of the orebody. If the source position is fixed at $\mathbf{s}$, the strongest asymptotic high-frequency contribution from the summation over $g$ geophone points is at the stationary point (Chen, 2004) that satisfies

$$
\operatorname{extrm}_{g}\left[\tau_{s x_{0} g}^{\mathrm{redu}}-\tilde{\tau}_{s x_{0} g}^{\mathrm{redu}}\right]=0 .
$$

The above condition is a special case of equation 19, except that equation 31 does not equal zero if the incorrect velocity is used and there is a geophone statics. There are two benefits here: The source static delays are eliminated by the subtraction $\tau_{s x_{0}}^{\mathrm{P}}-\tau_{s g}^{\mathrm{P}}$, and migration defocusing due to an incorrect velocity model is mitigated (see Appendix C).

As an example, Figure 15a shows the mispositioned image migrated from PS transmitted arrivals using a migration velocity with a $10 \%$ error. To mitigate the mispositioning error, the
Figure 15. Crosswell migration images of the PS transmissivity distribution using (a) standard and (b) reduced-time (see equation 29) migration. The input field data correspond to eight crosswell shot gathers with sources in the right well, each one shooting into 140 receivers on the left well; the migration velocity is computed by traveltime tomography. Panels (c and d) are similar except the PP reflection data are migrated. The dashed lines are the orebody boundaries estimated from well-log data and are consistent with the reduced migration images. The figure is taken from Sheley and Schuster (2003).
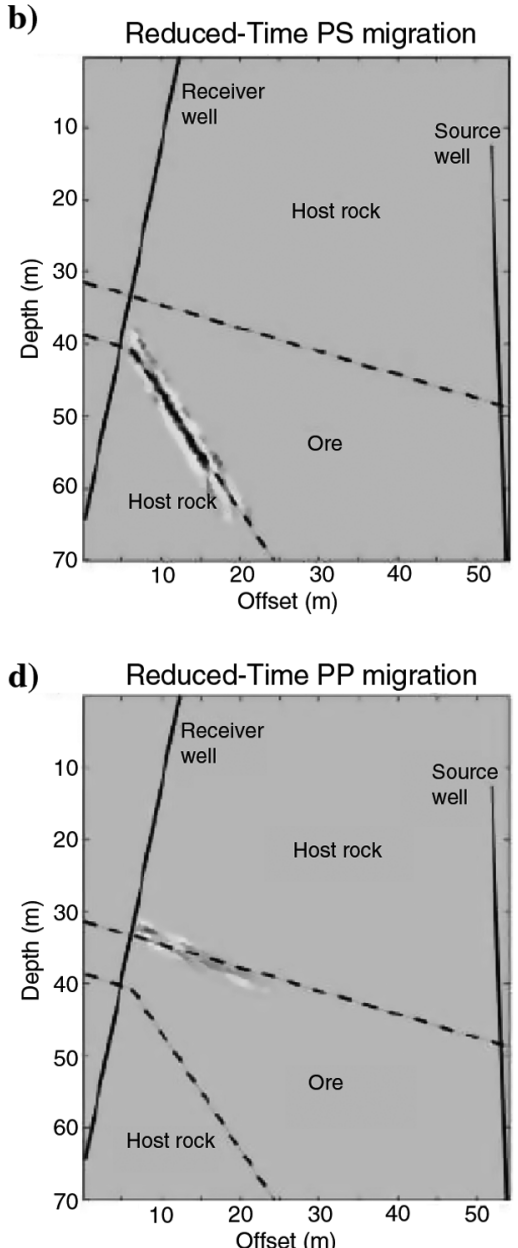
traveltime of the transmitted P-wave is picked and inserted into equation 29 to give the image shown in Figure 15. As predicted by theory (Sheley and Schuster, 2003), the mispositioning errors are largely reduced by the closure-phase condition in equation 31 .

\section{CONCLUSIONS}

We presented a review on geophysical applications of the general closure-phase equation 5, which is a weighted sum of traveltimes for specified events and is designed to eliminate unwanted phases in the data. It mathematically unifies many different processing methods independently developed in earthquake and exploration seismology. Some important benefits are elimination of static delays; reduced sensitivity of tomographic, migration, and hypocenter imaging to global velocity errors; equations for predicting far-offset traveltimes from shorter offset traveltimes; and S/N enhancement of noisy diffractions and refractions. The most important property is that, if the ray pairs are carefully selected, closure-phase imaging approximately redatums sources and/or receivers closer to the target of interest without knowing the velocity model.

Future developments of closure-phase imaging methods should test the target-oriented capabilities of reverse time migration, full wave inversion, and reflection traveltime tomography. It would be very important if closure phase plus full-waveform inversion can provide highly resolved velocity images of deep, not just shallow, hydrocarbon reservoirs as well as mantle geology.

\section{ACKNOWLEDGMENTS}

This work was supported in part by the King Abdullah University of Science and Technology and by the Utah Tomography and Modeling/Migration Consortium (http://utam.gg.utah.edu/) at the University of Utah.

\section{APPENDIX A}

\section{DIFFERENTIAL TRAVELTIMES AND MODEL COVARIANCE MATRIX}

The slowness resolution properties of differential traveltimes and absolute traveltimes are now analyzed with the slowness covariance matrix (Schuster et al., 1988; Menke, 1989) for the reflection traveltimes associated with the Figure A-1 ray diagrams. A compact derivation is given here that shows (1) the slowness uncertainties for differential tomography (DT) are larger than those for standard tomography (ST) and (2) to reduce the slowness uncertainties for DT, the difference in the raypath lengths of the differential ray pair should be large.

In Figure A-1, let ray $i$ be identified with the interface number upon which the ray reflects; let $l_{i}$ denote the segment length within any layer that ray $i$ visits (all layers are of equal thickness); let $t_{i}$ denote the total two-way traveltime belonging to ray $i$; let primed and unprimed versions of the above quantities be associated with far and near offsets, respectively; and let $s_{i}$ denote the slowness in the $i$ layer. The traveltime equations associated with rays $i$ and $i^{\prime}$ are therefore expressed as

$$
2 l_{i} \sum_{j=1}^{i} s_{j}=t_{i}+\epsilon_{i}
$$

$$
2 l_{i}^{\prime} \sum_{j=1}^{i} s_{j}=t_{i}^{\prime}+\epsilon_{i}^{\prime}
$$

Here, $\epsilon_{i}$ and $\epsilon_{i}^{\prime}$ represent independent random traveltime errors with zero mean and unity variance; i.e., $\operatorname{cov}\left(\epsilon_{i}, \epsilon_{j}\right)=\delta_{i j}, \operatorname{cov}\left(\epsilon_{i}^{\prime}, \epsilon_{j}^{\prime}\right)=$ $\delta_{i j}, \operatorname{cov}\left(\epsilon_{i}, \epsilon_{j}^{\prime}\right)=0$, where $\operatorname{cov}(\cdot, \cdot)$ denotes the covariance.

Introduce the cumulative slowness $u_{i}$, defined as

$$
u_{i}=\sum_{j=1}^{i} s_{j}, \quad \forall i=1, \ldots, n, \quad \text { and } \quad u_{0} \equiv 0
$$

where $n$ denotes the number of reflecting interfaces, for example, $n=4$ in Figure A-1. The inverse is given as

$$
s_{j}=u_{j}-u_{j-1}, \quad \forall j=1, \ldots, n
$$

In terms of $u_{i}$, the traveltime equations $\mathrm{A}-1$ and $\mathrm{A}-2$ can be rewritten as

$$
\mathbf{L}_{i} u_{i}=2\left[\begin{array}{c}
l_{i} \\
l_{i}^{\prime}
\end{array}\right] u_{i}=\left[\begin{array}{c}
t_{i} \\
t_{i}^{\prime}
\end{array}\right]+\left[\begin{array}{c}
\epsilon_{i} \\
\epsilon_{i}^{\prime}
\end{array}\right]
$$

where $\mathbf{L}_{i}$ is the $2 \times 1$ raypath matrix for the pair of near- and faroffset rays that reflect from the $i$ th interface. Observe here that, $\forall i=1, \ldots, n$, no equation contains more than one $u_{i}$. Consequently, the $u_{i}$ s are independent random variables (RVs). Moreover, the covariance of $u_{i}$ and $u_{j}$ is

$$
\operatorname{cov}\left(u_{i}, u_{j}\right)=\delta_{i j}\left[\mathbf{L}_{i}^{T} \mathbf{L}_{i}\right]^{-1}=\delta_{i j} \frac{1}{4\left(l_{i}^{2}+l_{i}^{\prime 2}\right)} .
$$

This says the covariance matrix of $\mathbf{u}=\left[u_{1}, \ldots, u_{n}\right]^{T}$ is diagonal, with the $i$ th diagonal element being $\frac{1}{4\left(l_{i}^{2}+l_{i}^{\prime 2}\right)}$. If the raypath segment lengths $l_{i}$ are reasonably large, then we can say that the variance of cumulative slowness in the $i$ th layer is desirably small for the standard traveltime equations.

To compare the above with that for differential traveltimes, we first design a subtraction strategy that achieves a specified goal. For example, choose differential traveltimes that eliminate source and receiver statics by subtracting equation $\mathrm{A}-1$ from $\mathrm{A}-2$ to give

$$
2\left(l_{i}^{\prime}-l_{i}\right) \sum_{j=1}^{i} s_{j}=t_{i}^{\prime}-t_{i}+\epsilon_{i}^{\prime}-\epsilon_{i},
$$

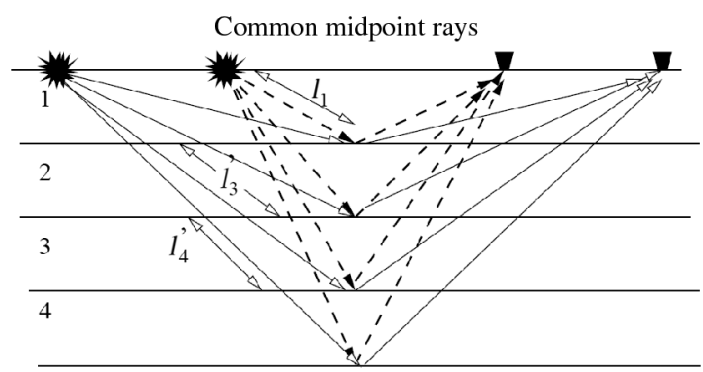

Figure A-1. Raypaths in a CMP geometry for two source-receiver pairs, assuming straight rays and layers with equal thickness. 
or

$$
2\left(l_{i}^{\prime}-l_{i}\right) u_{i}=t_{i}^{\prime}-t_{i}+\epsilon_{i}^{\prime}-\epsilon_{i}
$$

and thus,

$$
u_{i}=\overbrace{\frac{t_{i}^{\prime}-t_{i}}{2\left(l_{i}^{\prime}-l_{i}\right)}}^{\text {expectation }}+\overbrace{\frac{\epsilon_{i}^{\prime}-\epsilon_{i}}{2\left(l_{i}^{\prime}-l_{i}\right)}}^{\text {random error }}
$$

Because $\operatorname{var}\left(\epsilon_{i}^{\prime}-\epsilon_{i}\right)=2$, where $\operatorname{var}(\cdot)$ denotes the variance, the covariance of the resulting $u_{i}$ and $u_{j}$ is

$$
\operatorname{cov}\left(u_{i}, u_{j}\right)=\delta_{i j} \frac{1}{2\left(l_{i}^{\prime}-l_{i}\right)^{2}} .
$$

\section{Remark 1.}

This suggests, in comparison with equation A-6, that the uncertainties of cumulative slowness for DT are larger than those for ST. To reduce the uncertainties in the former case, given in equation A10 , we should try to maximize $\left|l_{i}^{\prime}-l_{i}\right|$. This can be accomplished by, for example, letting $l_{i}$ be associated with the smallest offset.

Last, we relate the covariance matrix of the cumulative slowness $\mathbf{u}$ to that of the slowness $\mathbf{s}=\left[s_{1}, \ldots, s_{n}\right]^{T}$. It follows from equation A-4 that

$$
\begin{aligned}
\operatorname{cov}\left(s_{i}, s_{j}\right)= & \operatorname{cov}\left(u_{i}-u_{i-1}, u_{j}-u_{j-1}\right), \\
= & \operatorname{cov}\left(u_{i}, u_{j}\right)+\operatorname{cov}\left(u_{i-1}, u_{j-1}\right) \\
& -\operatorname{cov}\left(u_{i}, u_{j-1}\right)-\operatorname{cov}\left(u_{i-1}, u_{j}\right) .
\end{aligned}
$$

We examine three cases:

1) When $|i-j|>1$, we have $\operatorname{cov}\left(s_{i}, s_{j}\right)=0$. The reason is twofold. First, independent RVs are uncorrelated (Papoulis, 1991, p. 154) and $u_{i}$ s are independent RVs. Second, when $|i-j|>1$, each term on the right-hand side (RHS) of equation A-12 is the covariance between two independent RVs.

2) When $|i-j|=1$, we have $\operatorname{cov}\left(s_{i}, s_{j}\right)=-\operatorname{cov}\left(u_{k}, u_{k}\right)$, where $k=\min (i, j)$. In this case, only one term on the RHS of equation A-12 is nonzero.

3) When $i=j$, we have $\operatorname{cov}\left(s_{i}, s_{i}\right)=\operatorname{cov}\left(u_{i}, u_{i}\right)+\operatorname{cov}\left(u_{i-1}, u_{i-1}\right)$, if $i>1$, and otherwise, $\operatorname{cov}\left(s_{1}, s_{1}\right)=\operatorname{cov}\left(u_{1}, u_{1}\right)$.

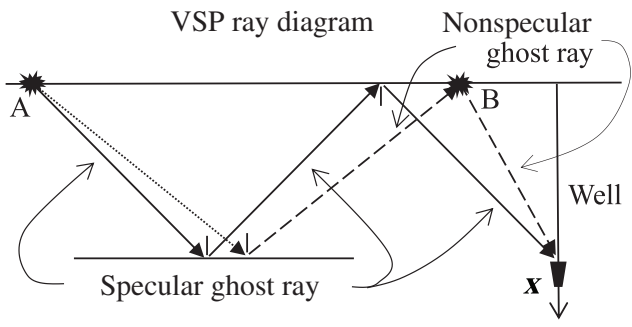

Figure B-1. Ray diagram for a walkaway VSP experiment where the sources are just below the free surface at locations $\mathbf{A}$ and $\mathbf{B}$ and the hydrophones are in the well. The specular ghost depicted as a solid ray honors Snell's law at each reflection point (denoted by short vertical bars), and the diffraction ray is denoted by dashed rays.
Therefore, the covariance matrix of $\mathbf{s}$ is tridiagonal. It is a simple exercise to verify that Remark 1 (regarding cumulative slowness) remains valid regarding slowness.

\section{APPENDIX B \\ STATIONARY CLOSURE PHASE AND RECIPROCITY THEOREMS}

The stationary closure-phase equations can be derived by applying stationary phase analysis to the reciprocity theorems of correlation and/or convolution types. We will demonstrate this for a three-point closure-phase equation associated with the VSP example in Figure B-1, but the derivation is extensible to stationary equations for an arbitrary number of points and arbitrary experimental geometries.

Assume the VSP model in Figure B-1 for harmonic-point sources just below the free surface and a background homogeneous velocity. Below this source-receiver configuration is a scattering medium sufficiently far away to satisfy the far-field approximation, where the wavenumber magnitude at the source location is given by $k$.

1) The Green's function $G(\mathbf{B} \mid \mathbf{A})$, characterizing the response at $\mathbf{B}$ in the frequency domain owing to a point impulse at $\mathbf{A}$, satisfies the far-field reciprocity equation of the correlation type (Snieder, 2004; Wapenaar, 2004; Wapenaar et al., 2005; Wapenaar and Fokkema, 2006):

$$
\mathfrak{I} \mathfrak{m}[G(\mathbf{B} \mid \mathbf{A})]=k \int G(\mathbf{A} \mid \mathbf{x}) G^{*}(\mathbf{B} \mid \mathbf{x}) \mathrm{d} x
$$

where the integration is over the area of overlying sources. For a medium characterized by distinct reflecting boundaries, the Green's function can be expressed as a sum of functions associated with individual events such as direct waves, primary reflections, and ghost reflections, e.g.,

$G(\mathbf{A} \mid \mathbf{x}) \approx G(\mathbf{A} \mid \mathbf{x})^{\text {direct }}+G(\mathbf{A} \mid \mathbf{x})^{\text {primary }}+G(\mathbf{A} \mid \mathbf{x})^{\text {ghost }}+\ldots$

This expression can be inserted into equation B-1 to give a product where the phase associated with different products can approximate the kinematics of a specified event. For example, the product of the direct arrival $G(\mathbf{B} \mid \mathbf{x})^{\text {direct }}$ and the ghost reflection $G(\mathbf{A} \mid \mathbf{x})^{\text {ghost }}$ functions gives

$$
\mathfrak{I m}\left[G(\mathbf{B} \mid \mathbf{A})^{\mathrm{SSP}}\right] \asymp k \int G(\mathbf{A} \mid \mathbf{x})^{\text {ghost }} G^{*}(\mathbf{B} \mid \mathbf{x})^{\text {direct }} \mathrm{d} x
$$

to obtain the virtual primary reflection for a SSP trace $G(\mathbf{B} \mid \mathbf{A})^{\text {SSP. }}$

2) Substituting the asymptotic Green's functions $G(\mathbf{A} \mid \mathbf{x})^{\text {ghost }}$ $G^{*}(\mathbf{B} \mid \mathbf{x})^{\text {direct }}=R(\mathbf{A}, \mathbf{B}, \mathbf{x})$ $\exp \left(i \omega\left[T_{A x}^{\text {ghost }}-T_{B x}^{\text {direct }}-T_{A B}^{\mathrm{SSP}}+T_{A B}^{\mathrm{SSP}}\right]\right)$ into the above formula yields 


$$
\begin{aligned}
{\left[G(\mathbf{B} \mid \mathbf{A})^{\mathrm{SSP}}\right] } & =k \exp \left(i \omega T_{A B}^{\mathrm{SSP}}\right) \int R(\mathbf{A}, \mathbf{B}, \mathbf{x}) \\
& \times \exp \left(i \omega[\overbrace{T_{A x}^{\text {ghost }}}^{\text {specular }}-\overbrace{\left(T_{B x}^{\text {direct }}+T_{A B}^{\mathrm{SSP}}\right)}^{\text {diffraction }}) \mathrm{d} x,\right.
\end{aligned}
$$

where $R(\mathbf{A}, \mathbf{B}, \mathbf{x})$ accounts for geometrical spreading effects. Stationary phase analysis (Snieder, 2004; Schuster, 2009) says that the leading contribution to the above integral is at the stationary point that satisfies the three-point stationarity condition, similar to equation 23 . In fact, there will be a very strong contribution to the integral from the wide range of sources postcritically offset from the receiver position. In this case, the asymptotic result is

$$
\mathfrak{I m}\left[G(\mathbf{B} \mid \mathbf{A})^{\mathrm{SSP}}\right]=C \exp \left(i \omega T_{A B}^{\mathrm{SSP}}\right)
$$

where $C$ is an asymptotic coefficient that also contains information about the geometrical spreading term $R(\mathbf{A}, \mathbf{B}, \mathbf{x})$.

The above procedure can be repeated by using different terms in equation B-2 to form a corresponding product in equation B-3. If this product enjoys a stationary point, which is possible for different events whose raypaths partly coincide with one another at $\mathbf{x}$ (Schuster, 2009), then it will give rise to a corresponding closurephase equation represented in general form by equation 19, and in specific form by equation 23 (Schuster, 2005a, 2005b), for example. There are many other examples of longer raypath events used to predict shorter ray events in the prediction of surface-related and interbed multiples (Jakubowicz, 1998; Sheng, 2001; Verschuur and Berkhout, 2005; Ramrez and Weglein, 2009). Similarly, the reciprocity equations of convolution type (Verschuur et al., 1992) can be used to predict long-ray events (free-surface-related multiples) by convolving and summing traces with shorter ray events (e.g., primaries).

Mallinson et al. (2011) and Bharadwaj et al. (2012) present the theory of supervirtual refraction interferometry where far-offset traces are generated by using the reciprocity theorem of correlation and convolution types. It can now be shown that the four-point closure-phase equation 25 is a direct consequence of applying stationary phase analysis to these two reciprocity equations. That is, the imaginary part of the virtual trace,

$$
\mathfrak{s} \mathfrak{m}\left[G\left(\mathbf{A} \mid \mathbf{x}^{\prime}\right)^{\text {virt-head }}\right]=k \int G(\mathbf{A} \mid \mathbf{x})^{\text {head }} G^{*}\left(\mathbf{x}^{\prime} \mid \mathbf{x}\right)^{\text {head }} \mathrm{d} x,
$$

is obtained, and it can be used to obtain $G\left(\mathbf{A} \mid \mathbf{x}^{\prime}\right)^{\text {virt-head }}$. This virtual Green's function is convolved (in the time domain) and integrated with the data to obtain the supervirtual trace,

$$
G(\mathbf{A} \mid \mathbf{B})^{\text {super-head }}=i k \int G\left(\mathbf{A} \mid \mathbf{x}^{\prime}\right)^{\text {virt-head }} G\left(\mathbf{B} \mid \mathbf{x}^{\prime}\right)^{\text {head }} \mathrm{d} x^{\prime} \text {. }
$$

The combination of these operations is given as

$$
\begin{aligned}
\mathfrak{I m}_{\mathfrak{m}}[ & \left.G(\mathbf{A} \mid \mathbf{B})^{\text {super-head }}\right] \\
= & \iint G(\mathbf{A} \mid \mathbf{x})^{\text {head }} G^{*}\left(\mathbf{x}^{\prime} \mid \mathbf{x}\right)^{\text {head }} G\left(\mathbf{B} \mid \mathbf{x}^{\prime}\right)^{\text {head }} \mathrm{d} x \mathrm{~d} x^{\prime}, \\
= & \exp \left(i \omega T_{A B}^{\text {head }}\right) \iint R\left(\mathbf{B}, \mathbf{A}, \mathbf{x}, \mathbf{x}^{\prime}\right) \\
& \times \exp \left(i \omega\left[T_{A x}^{\text {head }}-T_{A B}^{\text {head }}+T_{B x^{\prime}}^{\text {head }}-T_{x x^{\prime}}^{\text {head }}\right]\right) \mathrm{d} x \mathrm{~d} x^{\prime},
\end{aligned}
$$

where the asymptotic Green's functions have been used and $R\left(\mathbf{B}, \mathbf{A}, \mathbf{x}, \mathbf{x}^{\prime}\right)$ accounts for geometric spreading and the squared wavenumber.

If the set of subscripts $\left[A, x, x^{\prime}, B\right]$ in the exponent of equation B8 are replaced by the subscripts $[i, k, j, l]$, then the linear combination of traveltimes in the exponential is equal to the left-hand side of equation 25. For head waves, this sum of traveltimes is equal to zero as long as they correspond to head waves from the same refractor and the source and receiver locations are those depicted in Figure 10. Therefore, the values of $\mathbf{x}$ and $\mathbf{x}^{\prime}$ are stationary values (Snieder, 2004) over the postcritical integration limits and significantly contribute to the asymptotic form of this integral. It can be said that the four-point stationary closure phase (equation 25) follows a stationary phase analysis applied to the reciprocity equations of convolution and correlation types.

\section{APPENDIX C \\ ERROR ANALYSIS FOR PS MIGRATION WITH CLOSURE PHASE}

Consider the Figure 4a experiment in which the fundamental assumption is that the PS and P transmitted rays are almost coincident with one another from the source to the scatterer region at $\mathbf{x}_{j}$. The goal is to estimate the timing error in the traveltime imaging condition for the PS transmitted arrival in Figure 4a and compare it with that for reduced time migration associated with the Figure $4 d$ rays. The model is a scatterer at $\mathbf{x}_{j}$ embedded in a homogeneous background with a $\mathrm{P}$-wave velocity given by $v$; the ratio of $\mathrm{P}$ and $\mathrm{S}$ velocities is denoted as $\eta$ and the erroneous $\mathrm{P}$-wave migration velocity is given by $v^{\prime}=v+\delta v$. Here, the calculated imaging time $\tau_{i j k}^{\text {stnd }}$ for the standard migration of PS transmission waves is given as $\tau_{i j k}^{\mathrm{stnd}}=\tilde{T}_{i j}^{\mathrm{P}}+\tilde{T}_{j k}^{\mathrm{S}}=d_{i j} / v^{\prime}+\eta d_{j k} / v^{\prime}$, where $d_{i j}$ is the distance between the source at $\mathbf{x}_{i}$ and the trial scatterer point at $\mathbf{x}_{j}$. Correspondingly, the trial imaging time for reduced PS migration (Sheley and Schuster, 2003) is given by

$$
\begin{aligned}
\tau_{i j k}^{\mathrm{redu}} & =\tilde{T}_{i j}^{\mathrm{P}}+\tilde{T}_{j k}^{\mathrm{S}}-\tilde{T}_{i k}^{\mathrm{P}}+T_{i k}^{\mathrm{P}} \\
& =\frac{d_{i j}+\eta d_{j k}-d_{i k}}{v^{\prime}}+\frac{d_{i k}}{v},
\end{aligned}
$$

where $\tilde{T}_{i j}^{\mathrm{P}}$ is the calculated direct arrival of the P-wave, and the only term without the tilde is $T_{i k}^{\mathrm{P}}$ because it is the direct P-wave traveltime picked from the data. Denoting the actual slowness as $s=1 / v$ and approximating $1 / v^{\prime}=1 /(v+\delta v) \approx s-s^{2} \delta v$ for small $\delta v / v$ transforms the above equation into

$$
\tau_{i j k}^{\mathrm{redu}} \approx\left(d_{i j}+\eta d_{j k}\right) s+\epsilon^{\mathrm{redu}},
$$


where

$$
\epsilon^{\mathrm{redu}}=-s^{2} \delta v\left(d_{i j}+\eta d_{j k}-d_{i k}\right) .
$$

Compare this expression with the traveltime imaging condition for standard migration

$$
\tau_{i j k}^{\mathrm{stnd}}=\tilde{T}_{i j}^{\mathrm{P}}+\tilde{T}_{j k}^{\mathrm{S}} \approx\left(d_{i j}+\eta d_{j k}\right) s+\epsilon^{\mathrm{stnd}},
$$

where

$$
\epsilon^{\mathrm{stnd}}=-s^{2} \delta v\left(d_{i j}+\eta d_{j k}\right),
$$

which says that the magnitude of $\epsilon^{\text {redu }}$ must be less than that of $\epsilon^{\text {stnd }}$ for a given trace. Smaller errors in $\epsilon^{\text {redu }}$ mean better migration focusing than provided by standard migration.

\section{REFERENCES}

Bharadwaj, P., G. Schuster, I. Mallinson, and W. Dai, 2012, Theory of supervirtual refraction interferometry: Geophysical Journal International, 188, 263-273, doi: 10.1111/j.1365-246X.2011.05253.x.

Bharadwaj, P., X. Wang, G. Schuster, and K. McIntosh, 2013, Increasing the number and signal-to-noise ratio of OBS traces with supervirtual refraction interferometry and free-surface multiples: Geophysical Journal International, 192, 1070-1084, doi: 10.1093/gji/ggs087.

Chen, J., 2004, Specular ray parameter extraction and stationary-phase migration: Geophysics, 69, 249-256, doi: 10.1190/1.1649392.

Cornwell, T., 1989, The applications of closure phase to astronomical imaging: Science, 245, 263-269, doi: 10.1126/science.245.4915.263.

Cox, M. J., 1999, Static corrections for seismic reflection surveys: SEG Geophysical References Series 9.

Dai, W., T. Fei, Y. Luo, and G. T. Schuster, 2011, Super-virtual interferometric diffractions as guide stars: 81st Annual International Meeting, SEG, Expanded Abstracts, 3819-3823.

Derode, A., E. Larose, M. Tanter, J. De Rosny, A. Tourin, M. Campillo, and M. Fink, 2003, Recovering the Green's function from field-field correlations in an open scattering medium: Journal of the Acoustical Society of America, 113, 2973-2976, doi: 10.1121/1.1570436.

Docherty, P., 1992, Solving for the thickness and velocity of the weathering layer using 2-D refraction tomography: Geophysics, 57, 1307-1318, doi: 10.1190/1.1443198.

Douglas, A., 1967, Joint epicenter determination: Nature, 215, 47-48, doi: $10.1038 / 215047 \mathrm{a} 0$

Dziewonski, A. M., and D. L. Anderson, 1983, Travel times and station corrections for P waves at teleseismic distances: Journal of Geophysical Research: Solid Earth, 88, 3295-3314, doi: 10.1029/JB088iB04p03295.

Eubank, R. L., 1999, Nonparametric regression and spline smoothing, 2nd ed.: CRC Press.

Gerstoft, P., K. G. Sabra, P. Roux, W. Kuperman, and M. C. Fehler, 2006, Green's functions extraction and surface-wave tomography from microseisms in southern California: Geophysics, 71, no. 4, SI23-SI31, doi: 10 $.1190 / 1.2210607$

Halliday, D. F., A. Curtis, J. O. Robertsson, and D.-J. van Manen, 2007, Interferometric surface-wave isolation and removal: Geophysics, 72, no. 5, A69-A73, doi: 10.1190/1.2761967.

Hanafy, S. M., and G. T. Schuster, 2007, Target-oriented interferometric tomography for GPR data: Geophysics, 72, no. 3, J1-J6, doi: 10.1190/ 1.2437584

Hariharan, P., 2010, Basics of interferometry: Academic Press.

Jakubowicz, H., 1998, Wave equation prediction and removal of interbed multiples: 68th Annual International Meeting, SEG, Expanded Abstracts, $1527-1530$.

Jennison, R., 1958, A phase sensitive interferometer technique for the measurement of the Fourier transforms of spatial brightness distributions of small angular extent: Monthly Notices of the Royal Astronomical Society, 118, 276-284, doi: 10.1093/mnras/118.3.276.

Kaschwich, T., H. Gjøystdal, and I. Lecomte, 2011, Impact of diffraction on resolution of PSDM: 73rd Annual International Conference and Exhibition, EAGE, P384.

Keho, T. H., 1986, The vertical seismic profile: Imaging heterogeneous media: Ph.D. thesis, Massachusetts Institute of Technology.

Lay, T., and T. C. Wallace, 1995, Modern global seismology: Academic Press.
Luo, Y., Q. Liu, Y. E. Wang, and M. N. AlFaraj, 2006, Imaging reflectionblind areas using transmitted PS-waves: Geophysics, 71, no. 6, S241S250, doi: 10.1190/1.2358415.

Mallinson, I., P. Bharadwaj, G. Schuster, and H. Jakubowicz, 2011, Enhanced refractor imaging by supervirtual interferometry: The Leading Edge, 30, 546-550, doi: 10.1190/1.3589113.

Marsden, D., 1993a, Static corrections - A review, Part 1: The Leading Edge, 12, 43-49, doi: 10.1190/1.1436912.

Marsden, D., 1993b, Static corrections - A review, Part 2: The Leading Edge, 12, 115-120, doi: 10.1190/1.1436936.

Marsden, D., 1993c, Static corrections - A review, Part 3: The Leading Edge, 12, 210-216, doi: 10.1190/1.1436944.

Menke, W., 1989, Geophysical data analysis: Discrete inverse theory: Elsevier.

Michelini, A., and A. Lomax, 2004, The effect of velocity structure errors on double-difference earthquake location: Geophysical Research Letters, 31, L09602, doi: 10.1029/2004GL019682.

Nemeth, T., E. Normark, and F. Qin, 1997, Dynamic smoothing in crosswell traveltime tomography: Geophysics, 62, 168-176, doi: 10.1190/1.1444115.

Nikitchenko, A., D. Kiyashchenko, B. Kashtan, and V. Troyan, 2011, Diffraction imaging with cross-well seismic data: 73rd Annual International Conference and Exhibition, EAGE, B027.

Nolet, G., 1987, Seismic tomography: With applications in global seismology and exploration geophysics: Springer

Palmer, D., 1981, An introduction to the generalized reciprocal method of seismic refraction interpretation: Geophysics, 46, 1508-1518, doi: 10 $.1190 / 1.1441157$

Papoulis, A., 1991, Probability, random variables and stochastic processes, 3rd ed.: McGraw-Hill Companies.

Ramirez, A. C., and A. B. Weglein, 2009, Green's theorem as a comprehensive framework for data reconstruction, regularization, wavefield separation, seismic interferometry, and wavelet estimation: A tutorial: Geophysics, 74, no. 6, W35-W62, doi: 10.1190/1.3237118.

Rhodes, W. T., 2009, Closure phase and lucky imaging: Applied Optics, 48, A58-A62, doi: 10.1364/AO.48.000A58.

Schuster, G., 2005a, Fermat's interferometric principle for multiple reflection tomography: Geophysical Research Letters, 32, L12303, doi: 10 $.1029 / 2005$ GL022351.

Schuster, G. T., 2005b, Fermat's interferometric principle for target-oriented traveltime tomography: Geophysics, 70, no. 4, U47-U50, doi: 10.1190/1 .1997368.

Schuster, G. T., 2009, Seismic interferometry: Cambridge University Press.

Schuster, G. T., D. P. Johnson, and D. J. Trentman, 1988, Numerical verification and extension of an analytic generalized inverse for commondepth-point and vertical-seismic-profile traveltime equations: Geophysics, 53, 326-333, doi: 10.1190/1.1442466.

Sheley, D., 2001, Migration of transmitted arrivals: Master's thesis, University of Utah.

Sheley, D., and G. T. Schuster, 2003, Reduced-time migration of transmitted PS waves: Geophysics, 68, 1695-1707, doi: 10.1190/1.1620643.

Sheng, J., 2001, Migrating multiples and primaries in CDP data by crosscorrelation migration: 71 st Annual International Meeting, SEG, Expanded Abstracts, 1297-300

Sheng, J., , J. Yu, and G. T. Schusteret al., 2005, Tomostatics and closure phase residual statics applied to Saudi Aramco land data: 75th Annual International Meeting, SEG, Expanded Abstracts, 2229-2232.

Sherriff, R., 1991, Encyclopedic dictionary of exploration geophysics: SEG.

Snieder, R., 2004, Extracting the Green's function from the correlation of coda waves: A derivation based on stationary phase: Physical Review E, 69, 046610, doi: 10.1103/PhysRevE.69.046610.

Tsingas, C., B. El Marhfoul, S. Satti, and A. Dajani, 2012, Diffraction imaging as an interpretation tool: First Break, 30, 73-77doi: 10.3997/13652397.2011035

Verschuur, D., and A. Berkhout, 2005, Transforming multiples into primaries: Experience with field data: 75th Annual International Meeting, SEG, Expanded Abstracts, 2103-2106.

Verschuur, D. J., A. Berkhout, and C. Wapenaar, 1992, Adaptive surfacerelated multiple elimination: Geophysics, 57, 1166-1177, doi: 10 $1190 / 1.1443330$

Waldhauser, F., 2001, hypoDD: A program to compute double-difference hypocenter locations: USGS, Open file report, 01-113.

Waldhauser, F., and W. L. Ellsworth, 2000, A double-difference earthquake location algorithm: Method and application to the northern Hayward fault, California: Bulletin of the Seismological Society of America, 90, 13531368, doi: $10.1785 / 0120000006$.

Wapenaar, K., 2004, Retrieving the elastodynamic Green's function of an arbitrary inhomogeneous medium by cross correlation: Physical Review Letters, 93, 254-301, doi: 10.1103/PhysRevLett.93.254301.

Wapenaar, K., and J. Fokkema, 2006, Green's function representations for seismic interferometry: Geophysics, 71, no. 4, SI33-SI46, doi: 10.1190/1 .2213955 . 
Wapenaar, K., J. Fokkema, and R. Snieder, 2005, Retrieving the Green's function in an open system by cross correlation: A comparison of approaches: Journal of the Acoustical Society of America, 118, 27832786, doi: 10.1121/1.2046847.

Wiggins, R. A., K. L. Larner, and R. D. Wisecup, 1976, Residual statics analysis as a general linear inverse problem: Geophysics, 41, 922-938, doi: $10.1190 / 1.1440672$

Wohlleben, R., H. Mattes, and T. Krichbaum, 1991, Interferometry in radioastronomy and radar techniques: Springer.

Wright, C., 2008, Station corrections for the Kaapvaal seismic network: Statistical properties and relation to lithospheric structure: Physics of the Earth and Planetary Interiors, 167, 39-52, doi: 10.1016/j.pepi.2008.02.003.

Xue, Y., S. Dong, and G. T. Schuster, 2009, Interferometric prediction and subtraction of surface waves with a nonlinear local filter: Geophysics, $\mathbf{7 4}$ no. 1, SI1-SI8, doi: 10.1190/1.3008543.
Yu, J., 2000, Eliminating static shifts using the closure phase principle: Technical report, Utah Tomography and Modeling/Migration.

Zhang, H., and C. H. Thurber, 2003, Double-difference tomography: The method and its application to the Hayward fault, California: Bulletin of the Seismological Society of America, 93, 1875-1889, doi: 10 $.1785 / 0120020190$.

Zhou, M., 1999, Progress report on interferometric traveltime tomography: Technical report, Utah Tomography and Modeling/Migration.

Zhou, M., Z. Jiang, J. Yu, and G. T. Schuster, 2006, Comparison between interferometric migration and reduced-time migration of commondepth-point data: Geophysics, 71, no. 4, SI189-SI196, doi: 10.1190/1 .2213046 .

Zhou, M., and G. Schuster, 2000, Interferometric traveltime tomography: 70th Annual International Meeting, SEG, Expanded Abstracts, 21382141. 